\begin{tabular}{c|c|c|c|} 
SELECCIONES MATEMÁTICAS \\
Universidad Nacional de Trujillo \\
ISSN: $2411-1783$ (Online) \\
2021; Vol. 8(1): 173-195.
\end{tabular}

MATHEMATICS EDUCATION

\title{
Some Ideas on the Genesis of the Infinitesimal Calculus
}

\section{Algunas Ideas Sobre la Génesis del Cálculo Inifitesimal}

\author{
Alejandro Ortiz Fernández $\mathbb{}$
}

Received, Feb. 02, 2021

Accepted, Apr. 30, 2021

How to cite this article:

Ortíz A. Some Ideas on the Genesis of the Infinitesimal Calculus. Selecciones Matemáticas. 2021;8(1):173-195. http://dx.doi.org/10.17268/sel.mat.2021.01.16

\begin{abstract}
Via historical examples we will show some ideas and methods of mathematicians that contributed to the creation of the infinitesimal calculus.
\end{abstract}

Keywords . Calculus, exhaution, derivative, integral, quadrature, limit.

\section{Resumen}

Vía ejemplos históricos mostraremos algunas ideas y métodos de matemáticos que contribuyeron a la creación del cálculo infinitesimal.

Palabras clave. Cálculo, exhaución, derivada, integral, cuadratura, límite.

1. Ideas Precursoras en la Antigua Grecia. Sabemos que la matemática alcanzó un buen nivel de utilidad en las antiquísimas culturas egipcia y babilónica pero es en Grecia donde la matemática es creada como ciencia; el rigor en sus razonamientos fue característico en ellos. Vía sus argumentos matemáticos deseaban comprender el mundo físico. Llegaron a calcular las longitudes de curvas, áreas y volúmenes de muchos cuerpos geométricos, estudiaron las cónicas así como algunos problemas de máximos y mínimos. En este escenario destacamos las contribuciones de Arquímedes, Apolonio, Eratóstenes, Hiparco, Eudoxo de Cnido, Pappus. Los griegos se encontraron con el infinito, y con la noción de límite, lo que produjo una seria crisis en sus fundamentos. Estas ideas la trataron con el llamado "el método de exhaución", creado por Eudoxo, (408-355 A.C.), el que consiste en aproximar el área desconocida, que se desea encontrar, por áreas mayores y menores que se aproximan al área que se desea calcular. Este método se fundamenta en el siguiente principio:

"Dadas dos magnitudes desiguales, si de la mayor se resta una magnitud mayor que su mitad y de lo que queda otra magnitud mayor que su mitad y se repite continuamente este proceso, quedaría una magnitud menor que la menor de las magnitudes dadas". (ME)

Ejemplo 1.1 (La Cuadratura de la parábola. Arquímedes). Problema. "Encontrar el área de un segmento parabólico oblicuo ABC cortado por la cuerda $A C$, donde la tangente en B es paralela a AC."

Solución: El plan es construir una sucesión de figuras "agotadoras” $\left(A_{n}\right), n=1,2,3, \ldots$

Por construcción definimos $A_{1}=\triangle A B C$.

$A_{2}=\triangle A B C+\triangle A D B+\triangle B E C$, y así se construyen $A_{3}, A_{4}, \ldots, A_{n}$.

Lema 1.1. $|\triangle A B C|=4(|\triangle A D B|+|\triangle B E C|) \mid$, donde $|X|$ significa área de $X$.

Demostración:

$M N$ y $B O$ los tomamos como los ejes $x$ e $y$ de un sistema oblicuo de coordenadas. Las coordenadas de $E,\left(\xi, \frac{1}{2} y\right)$ satisface $\left(\frac{1}{2} y\right)^{2}=m \xi$, de donde $\xi=\frac{y^{2}}{4 m}$. Se tiene $G E=x-\xi=\frac{y^{2}}{m}-\frac{y^{2}}{4 m}=\frac{3}{4} \frac{y^{2}}{m}=\frac{3}{4} x=$ $\frac{3}{4} O B$.

*Sección Matemática. Pontificia Universidad Católica del Perú, Lima, Perú. (jorti z@pucp.edu •pe). 

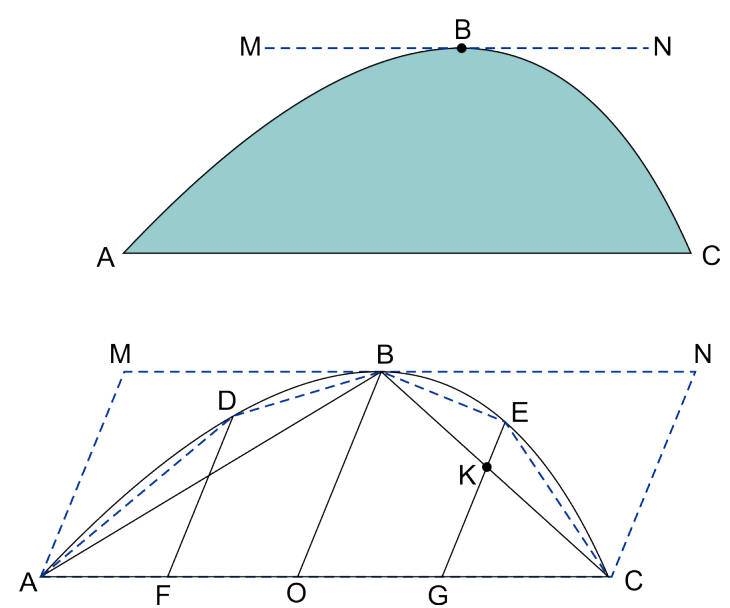

Figura 1.1: Idea Geométrica de la demostración del lema

Desde que $G K=\frac{1}{2} O B$ se obtiene $K E=E G-G K=\frac{3}{4} O B-\frac{1}{2} O B=\frac{1}{4} O B$, y $G K=2 K E$. Luego, $|\triangle C K G|=2|\triangle K C E|$ (tiene la misma altura) $=|\triangle B C E| ;|\triangle O B C|=4|\triangle G K C|=4|\triangle B C E|$. Análogamente, $\quad|\triangle A O B|=$ $|\triangle A B C|=|\triangle A O B|+|\triangle O B C|=4(|\triangle A B D|+|\triangle B C E|)$.

Si ponemos $\left|A_{1}\right|=|\Delta|$, entonces $\left|A_{2}\right|=|\Delta|+\frac{1}{4}|\Delta|,\left|A_{3}\right|=|\Delta|+\frac{1}{4}|\Delta|+\left(\frac{1}{4}\right)^{2}|\Delta|, \ldots,\left|A_{n}\right|=$ $|\Delta|+\frac{1}{4}|\Delta|+\ldots+\left(\frac{1}{4}\right)^{n-1}|\Delta| \ldots$

Lema 1.2. La sucesión $\left(A_{n}\right), n=1,2,3, \ldots$ "agota" el segmento parabólico, esto es, $S-\left|A_{n}\right|<\varepsilon$, donde $n=n(\varepsilon)$ y $S$ denota el área del segmento parabólico $A B C$.

Demostración: Consideremos el paralelogramo $A M N C$, según la figura, donde $A M / / N C / / B O$. Luego, $\left|A_{1}\right|=\frac{1}{2} S_{A M N C}$; pero $S<S_{A M N C}$, entonces $\frac{1}{2} S<\left|A_{1}\right|$ y $S-\left|A_{1}\right|<\frac{1}{2} S$. De esta manera se agota más de la mitad del área $S$; y así sucesivamente, las siguientes figuras agotaran más de la mitad de los correspondientes restos del área $S$. Luego se puede aplicar el método de exhaución $(M E)$ y concluir que $S-\left|A_{n}\right|<\varepsilon$, para $\varepsilon>0$ arbitrariamente pequeño.

Observemos que las figuras inscritas es una sucesión infinita. ¿Esta sucesión tiene límite?; si lo tiene, ¿Quién es? El siguiente resultado nos ayuda a clarificar esas cuestiones.

Teorema 1.1. Sea $S=|A|+|B|+|C|+|D|+|E|$ tal que

$|A|:|B|=|B|:|C|=|C|:|D|=|D|:|E|=4: 1$.

Entonces, $S=\frac{4}{3}|A|-\frac{1}{3}|E|$

Demostración: Por hipótesis, $|B|=\frac{1}{4}|A|,|C|=\frac{1}{4}|B|,|D|=\frac{1}{4}|C|,|E|=\frac{1}{4}|D|$.

Luego,

$$
\begin{aligned}
\frac{4}{3} S & =\frac{4}{3}(|A|+|B|+|C|+|D|+|E|)=\frac{4}{3}|A|+\frac{4}{3}(|B|+|C|+|D|+|E|) \\
& =\frac{4}{3}|A|+\frac{1}{3}(|A|+|B|+|C|+|D|) \\
& =\frac{4}{3}|A|+\frac{1}{3}(|A|+|B|+|C|+|D|+|E|)-\frac{1}{3}|E|,
\end{aligned}
$$

esto es, $\frac{4}{3} S=\frac{4}{3}|A|+\frac{1}{3} S-\frac{1}{3}|E|$.

Por tanto, $S=\frac{4}{3}|A|-\frac{1}{3}|E|$.

Nota. Este teorema puede ser extendido a cualquier número de sumandos.

Arquímedes hace uso de este teorema para obtener la igualdad:

$$
\left|A_{n}\right|=|\Delta|+\sum_{k=1}^{n-1} \frac{1}{4^{k}}|\Delta|=\frac{4}{3}|\Delta|-\frac{1}{3} \frac{|\Delta|}{4^{n-1}} .
$$

Luego, conjeturamos que si sigue con $n$ muy grandes $(n \rightarrow \infty)$, Arquímedes considera al sustraendo tan pequeño como se quiera y por tanto es despreciable, así $\left|A_{n}\right|$ se acerca a $S$, y Arquímedes concluye que $S=\frac{4}{3}|\Delta|$. 
Arquímedes (de Siracusa) representa el máximo de los genios matemáticos surgidos en la antigua Grecia, por la influencia de Euclides, alrededor del siglo III A.C. Como apreciamos, Arquímedes estuvo cerca el llegar al cálculo integral. Similar al milagro griego ocurrió alrededor del siglo XVII, época de gran esplendor en la historia de la matemática y que fue vital en la evolución de la ciencia y del inicio de la actual tecnología. Se llegó al siglo XVII con un progreso de la matemática gracias a contribuciones debidos a notables matemáticos como Pascal, Galileo, Fermat, Huygens, Roberval, Kepler, Cavalieri, Wallis, Gregory, Torriceli, Descartes, Barrow, entre otros, lo que permitió que en este siglo I. Newton y G. Leibniz crearan el cálculo infinitesimal.

\section{Miscelánea de ejemplos.}

Ejemplo 2.1 (Fermat y su Método de las Tangentes). Veamos los argumentos de Fermat para construir la tangente en un punto de la parábola siguiendo su modelo del método del máximo y mínimo. Veamos el siguiente gráfico. Según Fermat se va a determinar la subtangente $T Q$ a la parábola en el punto $P$. Observamos que los triángulos $T Q P$ y $T Q_{1} T_{1}$ son semejantes; luego se tiene

$$
\frac{T_{1} Q_{1}}{P Q}=\frac{T Q_{1}}{T Q} .
$$

Pero, desde que $P$ y $P_{1}$ están sobre la parábola $x=y^{2}$, se tiene también $\frac{V Q_{1}}{V Q}=\frac{P_{1} Q_{1}^{2}}{P Q^{2}}$ y además $P_{1} Q_{1}<T_{1} Q_{1}$. Luego tendremos $\frac{V Q_{1}}{V Q}<\frac{T Q_{1}^{2}}{T Q^{2}}$.

Pongamos $V Q=a$, la abscisa de $P$, la que es conocida pues $P$ es dado. Observamos que deseamos calcular la subtangente $T Q=x$. Bien, sea el incremento $Q Q_{1}=E$ y re-escribiendo la última desigualdad, tendremos $\frac{a+E}{a}<\frac{(x+E)^{2}}{x^{2}}$, la que es equivalente a $a x^{2}+E x^{2}<a x^{2}+2 a E x+a E^{2}$.

En esta etapa Fermat substituye esta desigualdad por una aproximación! a $x^{2}+E x^{2} \simeq a x^{2}+2 a E x+a E^{2}$,

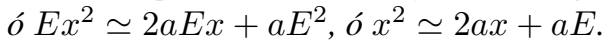

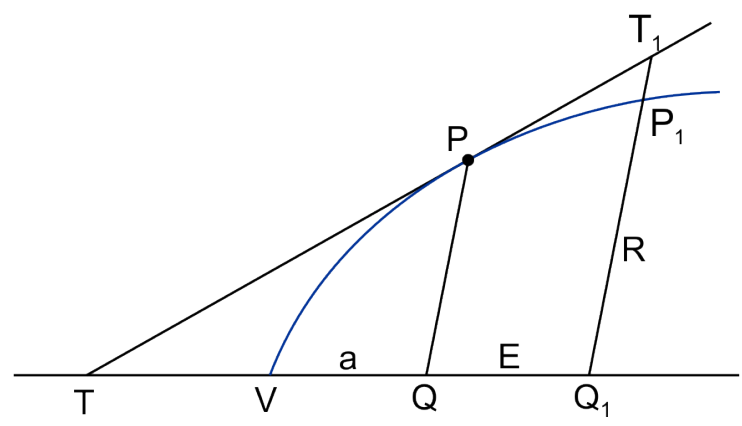

Figura 2.1: Método de Fermat sobre las tangentes

Si $E=0$ (idea de "limite"), $x \simeq 2 a$ ó "mejor" $x=2 a$. Así Fermat prueba que la subtangente es el doble de la abscisa del punto de tangencia $P$, es decir, se tiene $T V=V Q$, una idea ya conocida por los griegos. Así queda bien determinado el punto $T$, y por lo tanto la tangente $T P T_{1}$.

Observación 2.1. Este método de Fermat es ingenioso para la época en que fue hecho pero fue objeto de duras críticas por parte de Descartes lo que motivó que, en 1638, Fermat presentara un método más general para construir los tangentes.

\section{Ejemplo 2.2 (La Hoja de Descartes).}

En primer lugar veamos, brevemente, tal método general de Fermat.

Sea $P=(x, y)$ un punto sobre la curva $f(x, y)=0$. Objetivo: hallar la subtangente a respecto al punto P. Solución. Por semejanza de triángulos (ver figura) tenemos $z=\frac{y}{a}$ E. Por tanto, las coordenadas de un punto sobre la tangente, y próximo al punto de tangencia, es $\left(x+E, y+y \frac{E}{a}\right)$.

Ahora, (idea interesante) si E es muy pequeño! podríamos considerar este punto, que está sobre la tangente, como un punto sobre la curva (idea del límite!) y por tanto satisface la ecuación de la curva: $f\left(x+E, y\left(1+\frac{E}{a}\right)\right)=0$.

Ahora! Fermat usa su aguda intuición al considerar que para que esta última igualdad sea correcta, se debe poner $E=0$. Así, de la ecuación resultante se determina la subtangente " $a$ " en función de $x, y$ 


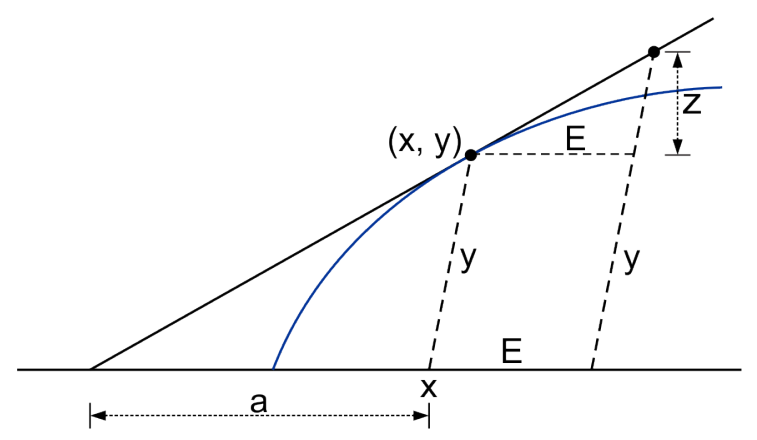

Figura 2.2: La hoja de Decartes

(coordenadas de P). Se establece que tal subtangente es equivalente a considerar:

$$
a=-y \frac{\frac{\partial f}{\partial y}}{\frac{\partial f}{\partial x}} .
$$

Aplicación a la hoja de Descartes. Fermat determinó la tangente para diversas notables curvas usando su método general, entre ellas a la hoja de Descartes, la que es definida por la función $x^{3}+y^{3}=n x y$. (d) Solución. Según el método se tiene

$$
(x+E)^{3}+\left(y\left(1+\frac{E}{a}\right)\right)^{3}-n y(x+E)\left(1+\frac{E}{a}\right)=0 \text {. }
$$

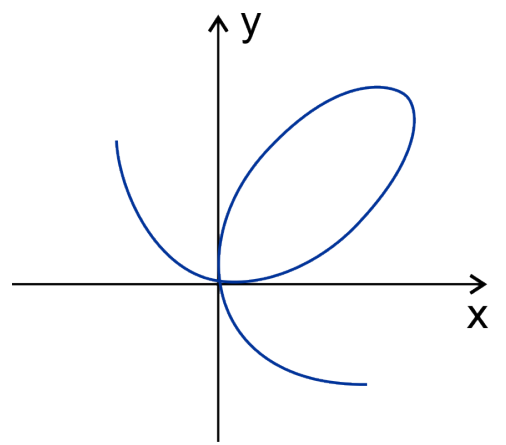

Hoja de Descartes para $n=3$

Figura 2.3: Hoja de Descartes $(n=3)$

Efectuando las potencias, agrupando en $E, E^{2}$ y $E^{3}$, se obtiene (considerando $(d)$ ):

$$
E\left(3 x^{2}+\frac{3}{a} y^{3}-\frac{n x y}{a}-n y\right)+E^{2}\left(3 x+\frac{3}{a^{2}} y^{3}-\frac{n y}{a}\right)+E^{3}\left(1+\frac{y^{3}}{a^{3}}\right)=0 .
$$

Ahora dividamos por $E(\neq 0)$, y luego "hagamos" $E=0$, para obtener $a=-y \frac{3 y^{2}-n x}{3 y^{2}-n y}$; así se ha encontrado "a", punto que con $P$ determinan a la tangente a la hoja de Descartes en el punto $P$. Nota. Por diferenciación implícita observamos que $\frac{\partial f}{\partial y}=3 y^{2}-n x, \frac{\partial f}{\partial x}=3 y^{2}-n y$ y así obtenemos (2.1).

\section{Ejemplo 2.3 (La Tangente según Barrow).}

Vía los métodos de algunos precursores del cálculo infinitesimal estamos viendo la evolución de la idea de límite. En esta dirección, Isaac Barrow (1630-1677) contribuyó con métodos e ideas que motivaron a su joven alumno I. Newton. Veamos.

Sea la curva $f(x, y)=0$ mostrada en la figura adjunta. Sea $P$ un punto sobre tal curva y $P R$ un incremento. El triángulo $P Q R$ es semejante al triángulo $P M N$; Barrow afirma que la pendiente $\frac{Q R}{P R}$ de la tangente es igual a la pendiente $\frac{P M}{M N}$ ! Intuitivamente observa que si el arco $P P^{\prime}$ es “muy pequeño”, entonces se le puede identificar con el segmento $P Q$ de la tangente sin cometer un "gran error". Así podemos 


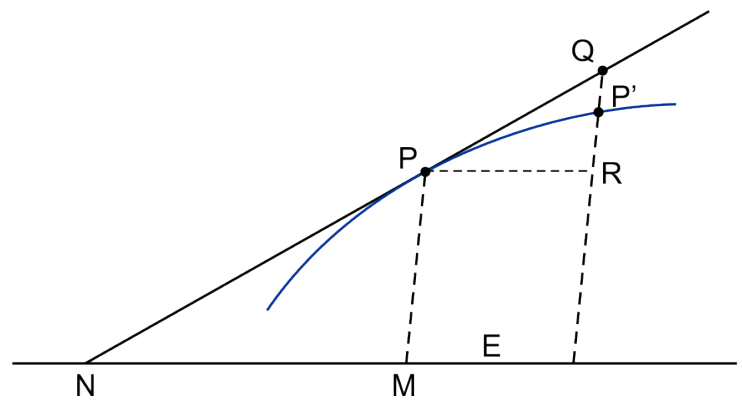

(b)

Figura 2.4: La tangente segun Barrow

“considerar" el triángulo $P R P^{\prime}$, donde $P P^{\prime}$ lo podemos considerar como un arco de curva ó como parte de la tangente. PRP' es llamado "triángulo característico".

Barrow, en sus "Lecciones Geométrica", calcula la tangente a una curva $f(x, y)=0$ en el punto $P=$ $(x, y)$, donde la ecuación es polinómica. Ver figura $(b)$.

Veamos la idea general, luego un caso concreto. Sea $P^{\prime}=(x+E, y+a)$ un punto de la curva cercano a $P$. Ahora substituyamos estas coordenadas en la ecuación $f(x, y)=0$.

Ahora Barrow nos dice: «Rechacemos todos los términos en los que no hay a ó E (porque se anulan unos a otros por la naturaleza de la curva); rechacemos todos los términos en los que a ó E están por encima de la primera potencia, o están multiplicados ambos (Porque, siendo infinitamente pequeños, no tienen valor en comparación con el resto). Luego de estas operaciones se calcula $\frac{a}{E}$, que es la pendiente de la curva en el punto $P \gg$.

Ejemplo concreto. Sea la curva $x^{3}+y^{3}=r^{3}$. Calcular la pendiente de la curva en el punto $P=(x, y)$.

Solución: Sea $P^{\prime}=(x+E, y+a)$ sobre la curva, cercano a $P(\operatorname{ver}(b))$. Luego $(x+E)^{3}+(y+a)^{3}=r^{3}$, de donde $x^{3}+3 x^{2} E+3 x E^{2}+E+E^{3}+y^{3}+3 y^{2} a+3 y a^{2}+a^{3}=r^{3}$. Pero, $x^{3}+y^{3}=r^{3}$; luego $3 x^{2} E+3 x E^{2}+E^{3}+3 y^{2} a+3 y a^{2}+a^{3}=0$.

Ahora se "eliminan" las potencias mayores que 1 de $E$ y $a$, obteniéndose $\frac{a}{E}=-\frac{x^{2}}{y^{2}}$, que es la pendiente de la curva en el punto $P=(x, y)$.

Nota. Observemos la analogía entre los métodos de Fermat y de Barrow para construir la tangente, métodos que "esconden" la idea de límite!

Ejemplo 2.4 (El Método de Exhaución. Continuación del Ejemplo 1).

La antigua cultura griega fue cuna de grandes pensadores; como hemos mencionado, una de sus principales contribuciones fue la solución del problema de la cuadratura del círculo usando el método de exhaución, idea que se remonta a la época de Sócrates y se atribuye a Eudoxo su creación; Arquímedes mejoró el método en su cuadratura de la parábola. Como sabemos, los griegos no tenían conceptos numéricos que les permitieran hacer corresponder a ciertas figuras geométricas, números que expresaran sus áreas (ó volúmenes), y de esta manera buscaron la razón entre algo por conocer con algo conocido. Así, Ejemplo 1, determinaron que $S=\frac{4}{3}|\Delta|$, donde $S$ es el área de un segmento parabólico y $|\Delta|$ es el área de un triángulo inscrito en él.

Remarcamos que tal método fue introducida a fin de evitar al infinito, idea que los griegos, desde los tiempos de Pitágoras, tenían cierto temor y no tuvieron un camino para explicar al infinito (ver Ejemplo 1). Esto fue una motivación para que los griegos (Eudoxo) desarrollaran una teoría de magnitudes y proporciones. Veamos algunos detalles.

Sea $X$ un área, una superficie o un volumen, que deseamos conocer ( $X$ está asociado a una figura geométrica). El método de exhaución permite asociar a X una magnitud conocida $A$ (asociada a alguna figura) del mismo tipo (por ejemplo, el área de un segmento parabólico es del mismo tipo que el área de un triángulo). La idea es considerar una sucesión monótona creciente $B_{n}$, inscrita en la figura " $X$ "; y una sucesión monótona decreciente $C_{n}$, circunscrita en la figura " $X$ ". de esta manera tenemos: $B_{n}<X<C_{n}, \forall n$. (i)

La "idea" ahora es que se verifica: «para cualquier $\varepsilon>0$ existe un número $N$ tal que $C_{N}-B_{N}<\varepsilon \gg$. (ii) (idea intuitiva que se tiene para un adecuado N). Ó también que se tiene: « para cualquier dos magnitudes $u$ y $v$, de misma clase, o $<v<u$, se tiene que existe un número $N$ tal que $\frac{C_{N}}{B_{N}}<\frac{u}{v}$. 》(iii) Además, se tiene: $\ll B_{n}<A<C_{n}, \forall n$. \(iv)

(una conclusión intuitiva). Entonces, $A=X$; es decir, el área ó volumen $X$ es igual a la magnitud $A$.

Nota. El argumento dado en el ejemplo 2.4 es intuitivo, esconde la idea de límite y de infinito, ideas en 
gestación en esa época y que causaron ciertas crisis en el pensamiento racional de los griegos. Observemos que el método solo puede ser usado si conocemos $A$. Hace falta (estaban cerca !) un método directo que verificara que

$$
\lim _{n \rightarrow \infty} B_{n}=\lim _{n \rightarrow \infty} C_{n}=X .
$$

Esto fue tarea de los matemáticos del siglo XVII. (Ver [5], pag. 31).

\section{Ejemplo 2.5 (Descartes y su Método de Trazar Tangentes).}

Sea $f(x, y)=0$ la ecuación de una curva y $P=\left(x_{1}, y_{1}\right)$ un punto de la curva, sobre el cual deseamos construir una tangente.

Solución. Sea $Q=\left(x_{2}, 0\right)$ un punto sobre el eje $x$. Tracemos la circunferencia con centro $Q$ y que pasa por $P$; su ecuación es:

$$
\left(x-x_{2}\right)^{2}+y^{2}=\left(x_{1}-x_{2}\right)^{2}+y_{1}^{2} .
$$

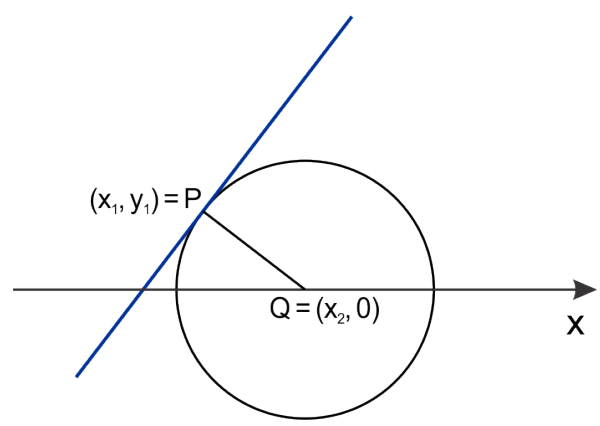

Figura 2.5: Método de descartes sobre la tangente

Ahora se elimina " $y$ " entre esta ecuación y $f=0$. Se obtiene así una ecuación en $x$, y las abscisas de puntos cuando la circunferencia corta a la curva. Ahora se determina $x_{2}$ así que tal ecuación en $x$ tenga un par de raíces iguales a $x_{1}$. Esta condición hace fijo $Q$ como la intersección del eje $x$ y la normal a la curva en $P$, desde que la circunferencia es ahora tangente a la curva dada en el punto $P$. Finalmente, tan pronto esta circunferencia es trazada, se puede construir la tangente deseada.

Caso concreto: "Construir la tangente a la parábola $y^{2}=4 x$ en el punto $(1,2)$."

Solución: Tenemos $\left(x-x_{2}\right)^{2}+y^{2}=\left(1-x_{2}\right)^{2}+4$.

Eliminando $y$ (se tiene $\left.y^{2}=4 x\right)$ tenemos $\left(x-x_{2}\right)^{2}+4 x=\left(1-x_{2}\right)^{2}+4$ ó

$x^{2}+2\left(2-x_{2}\right) x+\left(2 x_{2}-5\right)=0$. Ahora, la condición para que esta ecuación tenga dos raíces iguales es $\left(2-x_{2}\right)^{2}-\left(2 x_{2}-5\right)=0$, de donde $x_{2}=3$.

Por lo tanto es conocida la circunferencia con centro $(3,0)$ y que pasa por el punto $(1,2)$ de la parábola. Por lo tanto conocemos la pendiente del radio que une a estos puntos, que es perpendicular a la tangente a la parábola en el punto $(1,2)$. Luego conocemos la pendiente de la tangente, y de esta manera determinamos su ecuación.

\section{Ejemplo 2.6 (Máximos y Mínimos según Fermat).}

Sea $y=f(x)$ una función. Determinar sus valores máximos y mínimos.

Solución: Sea $x_{o}$ un punto del dominio de $f$. Observamos que cerca a $x_{o}$,

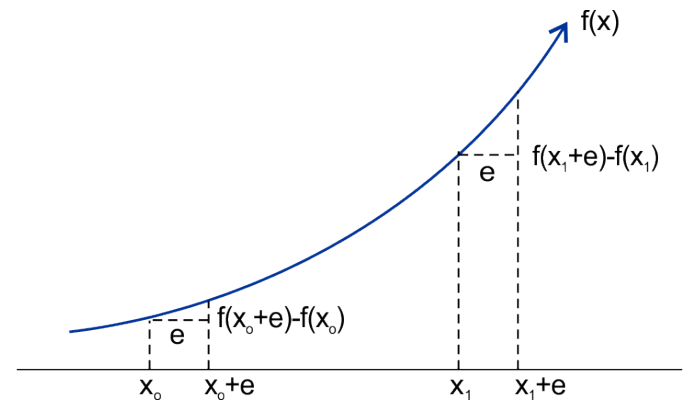

Figura 2.6: Valores críticos segun Fermat 
Cualquier cambio pequeño en este punto implica un pequeño cambio en el valor de $f\left(x_{0}\right)$ (no siempre es así.') y por ello es "despreciable". Se tiene $e=\left(x_{o}+e\right)-x_{o}=\left(x_{1}+e\right)-x_{1}$, luego las bases de los "triángulos" tienen la misma longitud pero los lados verticales tienen longitudes un poco diferentes (ó muy diferentes), ¿Por qué?... si tuviéramos una función continua ella podría casi "pararse” bruscamente y así la longitud del lado vertical de este triángulo puede ser muy grande. En relación a los "puntos críticos", donde la tangente es horizontal, veamos el argumento de Fermat para la función $f(x)=x^{3}-3 x^{2}$.

Bien, sea $x+e$ un punto cercano a $x$. Fermat considera que $f(x+e)-f(x)$ es "insignificante" y la hace igual a cero, esto es, $f(x+e)-f(x)=0$ y así se tiene $(x+e)^{3}-3(x+e)^{2}-x^{3}+3 x^{2}=0$, de donde ejecutando y simplificando se obtiene $3 x^{2} e+3 x e^{2}+e^{3}-6 x e-3 e^{2}=0$; dividiendo por e tenemos $3 x^{2}+3 x e+e^{2}-6 x-3 e=0$.

Ahora Fermat asume que " $e=0$ ", obteniendo $x^{2}-2 x=0$, de donde se obtiene que $x=0$ y $x=2$ son los valores críticos de $f(x)=x^{3}-3 x^{2}$.

Nota. Ahora que conocemos la idea de derivada sabemos que para calcular los valores críticos de una función se la deriva; así en el caso anterior, $f^{\prime}(x)=3 x^{2}-6 x$ y ponemos $f^{\prime}(x)=0$ (y la tangente en $x$ es paralela al eje $x$, y se "tiene" un máximo o un mínimo, aun cuando NO siempre!) cuyas soluciones son $x=0$ y $x=2$, como antes. Cuando Fermat considera $f(x+e)-f(x)=0$ ó $\frac{f(x+e)-f(x)}{e}=0$, el está considerando una "aproximación" ya que la derivada de $f(x)$ es el límite de este cociente cuando $e \rightarrow 0$; luego, cuando Fermat hace $e=0$, esto lo podríamos interpretar como " $e \rightarrow 0$ ". En la época de Fermat (siglo XVII) no existía la noción de derivada pero el germen de tal idea ya rondaba por tal época.

Ejemplo 2.7 (Fermat: Área bajo la curva $f(x)=x^{n}$ ).

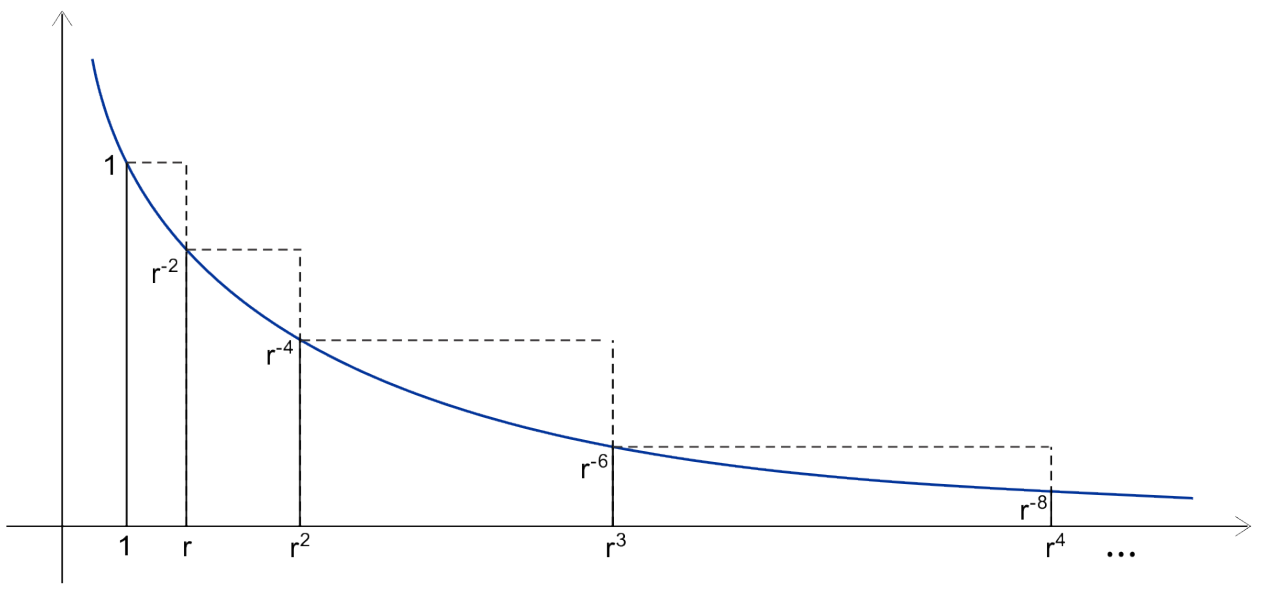

Figura 2.7: El área segun Fermat

Solución: Fermat discutió el caso $n=-2$ como guía y descubrir ideas para investigar el caso general. Veamos. Sea $r>1$ cualquier número real; para cada $j=1,2,3, \ldots$ considera la ordenada $\left(r^{j}\right)^{-2}=r^{-2 j}$, imagen del punto $x=r^{j}$ en el eje $x$ (ver figura adjunta). Así la región bajo la curva queda dividida en porciones que son "casi rectángulos", rectángulos que son obtenidos completando con los segmentos punteados (actualmente hacemos algo similar). Tenemos que el área del primer rectángulo es $(r-1) 1$, el área del segundo rectángulo es $\left(r^{2}-r\right) r^{-2}$, del tercero es $\left(r^{3}-r^{2}\right) r^{-4}, \ldots$, el área del j-ésimo rectángulo es:

$$
\left(r^{j}-r^{j-1}\right) r^{-2(j-1)}=r^{-j+1}(r-1)=\frac{r-1}{r^{j-1}} .
$$

Ahora se observa que $(r-1) 1,\left(r^{2}-r\right) r^{-2},\left(r^{3}-r^{2}\right) r^{-4}, \ldots,\left(r^{j}-r^{j-1}\right) r^{-2(j-1)}, \ldots$ es una progresión geométrica con razón $\frac{1}{r}$. Luego la suma de las áreas de tales rectángulos es: $S=(r-1)+(r-1) \frac{1}{r}+$ $(r-1)\left(\frac{1}{r}\right)^{2}+(r-1)\left(\frac{1}{r}\right)^{3}+\ldots$

$$
=(r-1)\left(1+\frac{1}{r}+\frac{1}{r^{2}}+\frac{1}{r^{3}}+\ldots\right)=(r-1) \frac{1}{1-\frac{1}{r}}=r .
$$


Por lo tanto, cuando $r$ se aproxima a $1(r \rightarrow 1)$ la suma de las áreas de los rectángulos se aproxima a 1 y los puntos $1, r, r^{2}, r^{3}, \ldots$ tienden a estar más densamente distribuidos en el eje $x$. De esta manera la unión de las áreas de los rectángulos se aproximan al área de la región bajo la curva $y=x^{-2}$. Así Fermat concluye que tal área es 1 .

Nota. Actualmente todo estudiante de matemáticas calcula tal área vía:

$$
\int_{1}^{\infty} x^{-2} d x=1
$$

Además, en el método de Fermat está el germen de la integral de Cauchy y la de Riemann.

Ejemplo 2.8 (Los Indivisibles de Cavalieri). Antecedentes. El punto de partida es Arquímedes (ver Ejemplo 1), quien fue desconocido por buen tiempo. En los siglos XVI y XVII Simón Stevin y Luca Valerico trataron de evitar el método de exhaución pasando directamente al límite. Posteriormente otros matemáticos como Kepler, Torricelli, Wallis, Cavalieri, Pascal y Otros, fueron herederos de la obra del genial matemático griego. Johann Kepler (1571-1630) merece algunos comentarios pues habría de contribuir con ideas en pro del desarrollo del cálculo infinitesimal; así, por ejemplo, el considera a un sólido como compuesto de infinitos elementos infinitesimales cuyos volúmenes son conocidos o factibles de calcularse ("Nueva Geometría Sólida de los Barriles de Vino”). Así, lo esencial de la idea de Kepler es la identificación de las áreas y volúmenes de cuerpos físicos con la suma de un número infinito de elementos infinitesimales. Por ejemplo, el círculo puede considerarse como la suma de un número infinito de triángulos; un área se podía considerar como suma de líneas.

En tal escenario tenemos la contribución de Bonaventura Cavalieri (1578-1647), un alumno de Galileo, quien también es considerado un precursor del cálculo infinitesimal. Conoció los trabajos de los griegos, estuvo en el camino seguido por Arquímedes. Fue influido por Galileo y por Kepler para investigar al cálculo, en particular al infinito y a los "indivisibles" en donde están los gérmenes del cálculo infinitesimal, ideas que fueron investigadas por Eudoxo y Arquímedes. Cavalieri creó un método de integración basado en los indivisibles, una idea que él no define pero que asume que son objetos con una dimensión menor al continuo del que forma parte. Por ejemplo, los puntos son los indivisibles de las líneas, las líneas son indivisibles de las figuras planas, estas lo son de los sólidos. Observamos que esta idea es intuitiva y Cavalieri la usó para explicar una técnica para calcular áreas y volúmenes.

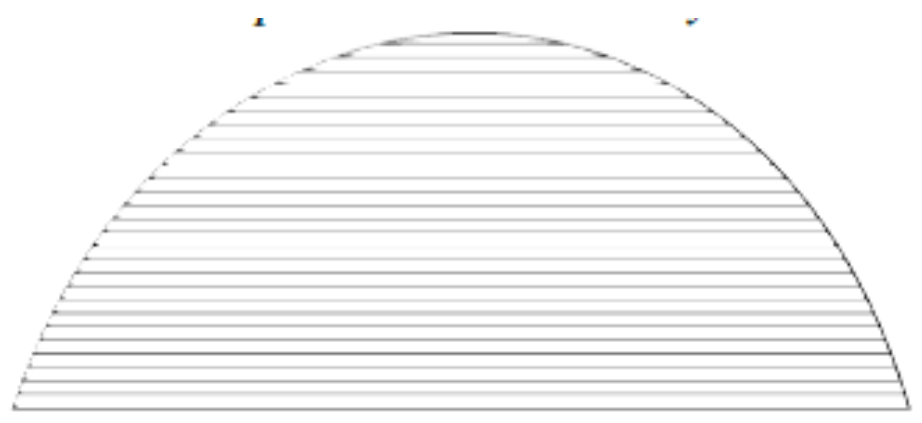

Figura 2.8: Las lineas son los indivisibles de las figuras planas

Concretamente, veamos como Cavalieri trabaja con indivisibles de la forma $x^{P}$. Para ello establece la propiedad:

$$
\frac{\sum_{1}^{n} x^{P}}{n c^{P}}=\frac{1}{p+1}
$$

donde $x$ va de 0 a c."

En efecto:

(i) si $p=1$, se tiene $\frac{\sum_{1}^{n} x}{n c}=\frac{1}{2}$.

De esta manera, la suma $\sum_{1}^{n} x$ de $n$ indivisibles de la forma $x$, donde $x$ va de 0 a c, es a la suma de indivisibles iguales a c como 1 es a 2. De esta manera Cavalieri afirma que los indivisibles de un triángulo equivale a tantos indivisibles iguales a la mitad de la base, esto es, el triángulo es equivalente al paralelogramo de igual base y con mitad de altura. 
(ii) si $p=2$, se tiene

$$
\frac{\sum_{1}^{n} x^{2}}{n c^{2}}=\frac{1}{3} \text {. }
$$

De esta manera la suma de los cuadrados de los segmentos de un triángulo es a la suma de tantos cuadrados de la base como segmentos, como 1 es a 3. Así, la pirámide es la tercera parte del prisma de igual base y altura

(iii) Si $p=3$ Cavalieri comprueba que se tiene:

$$
\frac{\sum_{1}^{n} x^{3}}{n c^{3}}=\frac{1}{4} \text {. }
$$

(iv) Para $p=4, \frac{\sum_{1}^{n} x^{4}}{n c^{4}}=\frac{1}{5}, \ldots$

Finalmente, Cavalieri induce que en general se tiene

$$
\frac{\sum_{1}^{n} x^{p}}{n c^{p}}=\frac{1}{p+1} .
$$

Nota. Actualmente tenemos: Si $p \neq-1$,

$$
\int_{0}^{c} x^{p} d x=\frac{c^{p+1}}{p+1}=\lim _{n \rightarrow \infty} \frac{c n c^{p}}{n(p+1)}=\lim _{n \rightarrow \infty} \frac{c \sum_{1}^{n} x^{p}}{n} .
$$

Principio de Cavalieri: "Si dos figuras planas tiene la misma altura y si las líneas producidas por líneas paralelas a las bases y a igual distancia de ellas están siempre en la misma razón, entonces las figuras dadas también están en la misma razón".

Por ejemplo, tenemos:
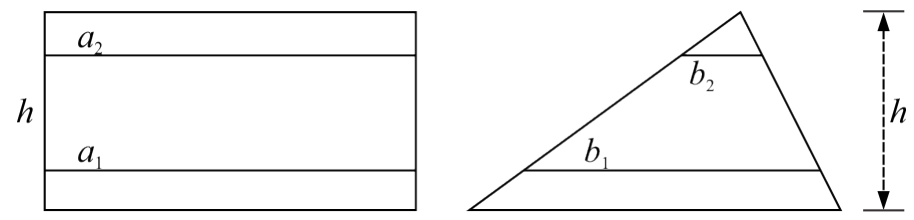

Figura 2.9: El principio de Cavalieri

Si $\frac{a_{1}}{b_{1}}=\frac{a_{2}}{b_{2}}=r$, entonces $\frac{\square}{\triangle}=r$.

Nota. Un análogo principio existe para el caso de dos sólidos.

Área de la Elipse. Sea una elipse de semi-ejes a y b, $a>b$; y una circunferencia concéntrica con la elipse y radio a. Así tenemos las formulas, respectivamente,

$\frac{x^{2}}{a^{2}}+\frac{y^{2}}{b^{2}}=1 \mathrm{y} x^{2}+y^{2}=a^{2}$. Despejando y en estas ecuaciones, obtenemos $y=\frac{b}{a}\left(a^{2}-x^{2}\right)^{\frac{1}{2}} \mathrm{y}$ $y=\left(a^{2}-x^{2}\right)^{\frac{1}{2}}$. Considerando que estas y’s son diferentes tenemos $\frac{y_{e}}{y_{c}}=\frac{b}{a}$. Luego la razón entre dos cuerdas verticales correspondientes de la elipse y de la circunferencia es $\frac{b}{a}$; por el principio de Cavalieri se tiene $\frac{\mid \text { elipse } \mid}{\mid \text { circulo } \mid}=\frac{b}{a}$. 


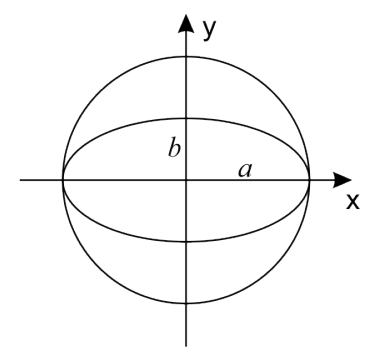

Figura 2.10: Área de la elipse

De esta manera: área de la elipse $=\frac{b}{a}$ (área del circulo) $=\frac{b}{a} \pi a^{2}=\pi a b$.

Ejemplo 2.9 (Wallis, Otro Precursor del Cálculo).

$$
\int_{0}^{1} x^{2} d x=\frac{1}{3}
$$

Antecedentes. Arquímedes usó el método de exhaución para evitar al infinito trabajando directamente en la figura geométrica. Posteriormente, Cavalieri reemplaza a la figura por "infinitos" indivisibles, elementos que tienen una dimensión menor que la figura, pero Cavalieri no precisa que es un "indivisible"; además, el uso del infinito es no claro.

En este escenario tenemos también John Wallis (1616-1703), quien es considerado el más importante matemático anterior a Newton. En 1656 publicó dos libros, uno sobre geometría analítica y otro sobre el cálculo infinitesimal: las dos teorías más importantes de la época. Veamos que en general se tiene

$$
\int_{0}^{a} x^{k} d x=\frac{a^{k+1}}{k+1}(k \neq-1)
$$

Cavalieri llega a esta fórmula vía sus indivisibles y de una manera un tanto intuitivo; Wallis deja tal método; en vez de trabajar con indivisibles lo hace con valores numéricos asociados a tales indivisibles. Wallis observa que:

$$
\begin{aligned}
\frac{0^{2}+1^{2}}{1^{2}+1^{2}}=\frac{1}{2}=\frac{1}{3}+\frac{1}{6}, \quad & \frac{0^{2}+1^{2}+2^{2}}{2^{2}+2^{2}+2^{2}}=\frac{5}{12}=\frac{1}{3}+\frac{1}{12}, \\
\frac{0^{2}+1^{2}+2^{2}+3^{2}}{3^{2}+3^{2}+3^{2}+3^{2}}=\frac{7}{18}=\frac{1}{3}+\frac{1}{18}, & \frac{0^{2}+1^{2}+2^{2}+3^{2}+4^{2}}{4^{2}+4^{2}+4^{2}+4^{2}+4^{2}}=\frac{3}{8}=\frac{1}{3}+\frac{1}{24},
\end{aligned}
$$

Inductivamente Wallis asume que $L \equiv \frac{0^{2}+1^{2}+2^{2}+3^{2}+\ldots+n^{2}}{n^{2}+n^{2}+n^{2}+n^{2}+\ldots+n^{2}}=\frac{1}{3}+\frac{1}{6 n}$.

Por tanto, $\lim _{n \rightarrow \infty} L=\frac{1}{3}$.

Se observa que la razón de las sumas de los cuadrados de los indivisibles en dos figuras (deseamos calcular $\left.\int_{0}^{1} x^{2} d x\right)$ sería:

para dos indivisibles, la razón sería $\frac{0^{2}+1^{2}}{1^{2}+1^{2}}=\frac{1}{2}$; para tres indivisibles $\frac{0^{2}+1^{2}+2^{2}}{2^{2}+2^{2}+2^{2}}=\frac{5}{12}$, ..., para $n+1$ indivisibles $\frac{0^{2}+1^{2}+2^{2}+\ldots+n^{2}}{n^{2}+n^{2}+n^{2}+\ldots+n^{2}}=\frac{1}{3}+\frac{1}{6 n}$.

Veamos los argumentos de Wallis para encontrar el área bajo la parábola $y=x^{2}$ de $x=0$ a $x=a$.

Wallis imaginó la región compuesta de $n$ angostos rectángulos verticales, cada uno con base $\frac{a}{n}$.

La razón de la suma de las áreas de esos rectángulos con las áreas de los rectángulos circunscritos se comporta como:

(así sale la relación $L, !)$.

$$
\frac{\left(\frac{0 a}{n}\right)^{2}+\left(\frac{1 a}{n}\right)^{2}+\left(\frac{2 a}{n}\right)^{2}+\ldots+\left(\frac{n a}{n}\right)^{2}}{a^{2}+a^{2}+a^{2}+\cdots+a^{2}}=\frac{0^{2}+1^{2}+2^{2}+\ldots \cdot+n^{2}}{n^{2}+n^{2}+n^{2}+\cdot+n^{2}}
$$

Esto induce a Wallis a concluir que:

$$
\frac{\text { area } o a b}{\text { area } o a b c}=\lim _{n \rightarrow \infty} \frac{0^{2}+1^{2}+2^{2}+\ldots \cdot+n^{2}}{n^{2}+n^{2}+n^{2}+\cdot+n^{2}}=\frac{1}{3} .
$$

Observemos que, en notación moderna, este resultado es equivalente a 


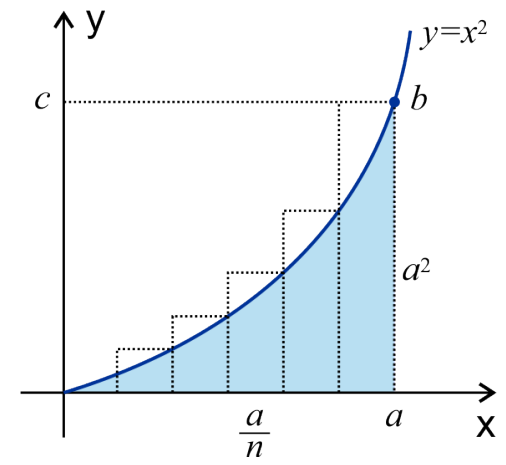

Figura 2.11: Método de Wallis para calcular el área bajo la curva $y=x^{2}$

$$
\frac{\int_{0}^{a} x^{2} d x}{a^{3}}=\frac{1}{3} .
$$

Así Wallis es inducido a concluir que $\int_{0}^{a} x^{k} d x=\frac{a^{k+1}}{k+1}, k$ entero positivo.

En particular, $\int_{0}^{1} x^{2} d x=\frac{1}{3}$.

Ejemplo 2.10 (J. Wallis). Calcular

(i) $\lim \frac{1^{3}+2^{3}+3^{3}+\ldots+n^{3}}{n^{3}+n^{3}+n^{3}+\ldots+n^{3}}$

(ii) $\int_{0}^{a} x^{3} d x$

Solución. (i) Se tiene $\frac{0^{3}+1^{3}}{1^{3}+1^{3}}=\frac{1}{2}=\frac{1}{4}+\frac{1}{1 \cdot 4}, \quad \frac{0^{3}+1^{3}+2^{3}}{2^{3}+2^{3}+2^{3}}=\frac{9}{24}=\frac{1}{4}+\frac{1}{2 \cdot 4}$,

$\frac{0^{3}+1^{3}+2^{3}+3^{3}}{3^{3}+3^{3}+3^{3}+3^{3}}=\frac{36}{108}=\frac{1}{4}+\frac{1}{12}=\frac{1}{4}+\frac{1}{3 \cdot 4}$,

$\frac{0^{3}+1^{3}+2^{3}+3^{3}+4^{3}}{4^{3}+4^{3}+4^{3}+4^{3}+4^{3}}=\frac{100}{320}=\frac{1}{4}+\frac{1}{16}=\frac{1}{4}+\frac{1}{4 \cdot 4}, \ldots$,

luego $L=\lim _{n \rightarrow \infty} \frac{1^{3}+2^{3}+3^{3}+\ldots+n^{3}}{n^{3}+n^{3}+n^{3}+\ldots+n^{3}}=\lim _{n \rightarrow \infty}\left(\frac{1}{4}+\frac{1}{n \cdot 4}\right)=\frac{1}{4}$.

(ii). El intervalo $[0, a]$ es dividido en $n$ sub intervalos iguales, cada uno de base $\frac{a}{n}$.

Entonces tenemos:

$$
\begin{aligned}
\frac{\int_{0}^{a} x^{3} d x}{a^{4}} & =\lim _{n \rightarrow \infty} \frac{\left(\frac{0 a}{n}\right)^{3}+\left(\frac{1 a}{n}\right)^{3}+\left(\frac{2 a}{n}\right)^{3}+\ldots+\left(\frac{n a}{n}\right)^{3}}{a^{3}+a^{3}+a^{3}+\ldots+a^{3}} \\
& =\lim _{n \rightarrow \infty} \frac{0^{3}+1^{3}+2^{3}+\ldots+n^{3}}{n^{3}+n^{3}+n^{3}+\ldots+n^{3}}=\frac{1}{4}
\end{aligned}
$$

Luego, $\int_{0}^{a} x^{3} d x=\frac{a^{4}}{4}$

Nota. Wallis encontró una similar fórmula para hallar el valor de $\int_{0}^{1} x^{\frac{m}{n}} d x$.

Ilustremos la idea calculando $\int_{0}^{1} x^{\frac{1}{2}} d x$. Veamos.

Sabemos que $\int_{0}^{1} x^{2} d x=\frac{1}{3}$.

Si $y=x^{2}, x=\sqrt{y} ; x=0$ implica $y=0, x=1$ implica $y=1$.

Luego, $\int_{0}^{1} y^{\frac{1}{2}} d y=\left.\frac{2}{3} y^{\frac{3}{2}}\right|_{0} ^{1}=\frac{2}{3}$.

Ejemplo 2.11 (Barrow. El Teorema Fundamental del Cálculo).

Las ideas de derivada y de integral descansan en la noción de limite; ¿existe alguna relación entre estos límites? Históricamente los problemas de trazar tangentes y el de hallar cuadraturas preocupó a matemáticos desde la época de los antiguos griegos. El desarrollo de la física por Galileo, Oresme, Torricelli, ...., va vislumbrando el carácter inverso de las operaciones de cuadratura (conocida la velocidad, se conoce el 


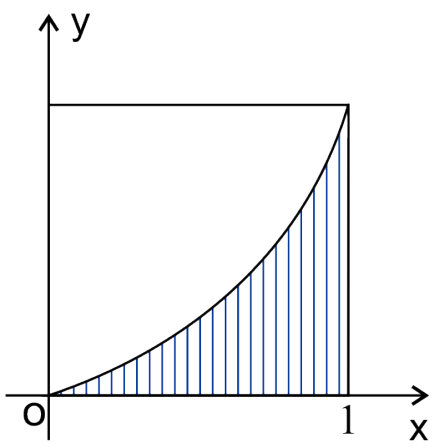

Figura 2.12: $\int_{0}^{1} x^{2} d x=\frac{1}{3}$

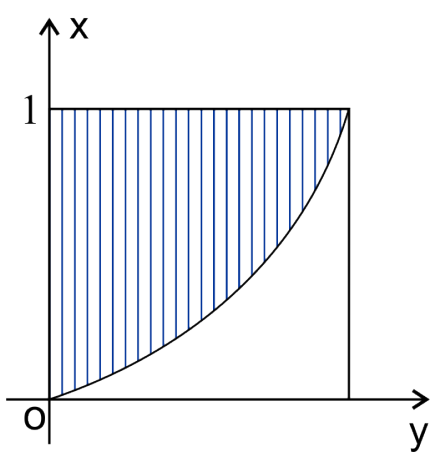

$\int_{0}^{1} y^{\frac{1}{2}} d y=\frac{2}{3}$

espacio) con las de construcción de tangentes (conocido el espacio, se conoce la velocidad). Con notación actual, se tiene:

$$
\int_{0}^{t_{o}} v(t) d y=\left.S\left(t_{o}\right) \quad y \quad \frac{d s}{d t}\right|_{t=t_{o}}=v\left(t_{o}\right) .
$$

En el siglo XVII, Barrow estuvo cerca de descubrir que el problema de la tangente y el de la cuadratura son inversas una de otra. Por buen tiempo estos dos problemas iban por rutas diferentes; las cuadraturas fueron investigados por Arquímedes en la lejana Grecia y las tangentes mucho después. El método geométrico usado por Barrow es un tanto elaborado, difícil de comprender a un lector actual. Tal enfoque no le permitió llegar al teorema fundamental, pero sus ideas fueron motivadoras para que su brillante alumno Newton y Leibniz, llegaron a formular tal relación dentro de una teoría coherente. Por razones históricas vamos a presentar la ruta seguida por Barrow (posiblemente con la misma notación usada por él). Ver [4] para otros detalles.

Teorema 2.1 ( Barrow). (Ver figura abajo). $y=f(x)$ representa a la curva ZGE y $g(x)$ a la curva VIF. $V D$ es el eje de las abscisas. La cantidad $g(x)$ va a representar el valor del área bajo la gráfica de $f(x)$ comprendida entre los puntos $V$ y $x$.

Dado el punto de abscisa $D$, entonces se tiene:

[pendiente de la tangente a $y=g(x)$ en el punto

$$
F=(D, g(D))=(f(D)=D E)
$$

Observación. Si observamos con atención, (2.11) es, en el lenguaje actual, equivalente a $(\bullet) \ldots \frac{d}{d_{X}} \int_{0}^{x} f(t) d t=$ $f(x)$; ver también (2.10).

Demostración:

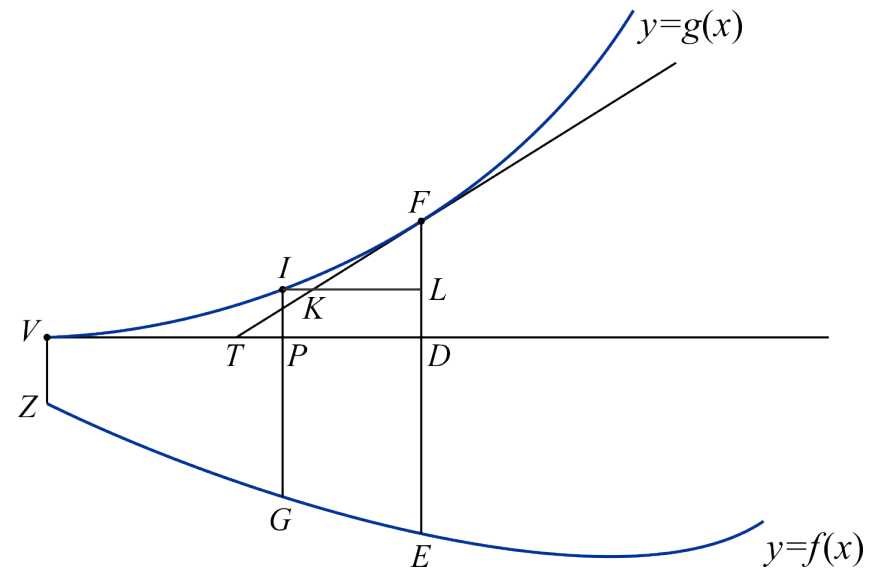

Figura 2.13: Teorema Fundamental del Cálculo segun Barrow 
Tracemos una recta $F T$ que corta a la recta $V D$ en el punto $T$ y tal que $\frac{D F}{T D}=f(D)(=D E)$. (Se asume parte de la tesis)

Luego queda por probar: $F T$ es precisamente la tangente a la curva $y=g(x)$ en el punto $F$. En efecto, con este objetivo verificaremos que la distancia horizontal $K L$, de cualquier punto $L$ de la recta $E F$ a la recta $F T$, es menor que la distancia $I L$ de dicho $L$ a la curva $y=g(x)$.

Un resultado análogo se obtendría si el punto $I$ estuviera a la derecha de $F$. entonces se habría probado que la recta $F T$ queda siempre debajo de la curva $y=g(x)$, es decir, $F T$ es la tangente a $y=g(x)$ en el punto $F$.

Bien, tenemos que $\frac{F L}{K L}=\frac{D E}{T D}=D E$. Por otra parte, se ha asumido que: área $V D E Z=F D$, área $V P G Z=P I=L D$ y que, área $P D E G=F D-L D=F L$. Pero, desde que área $P D E G<$ área rectángulo $P D \cdot D E$, se tiene $F L<P D \cdot D E$. Luego, $D E>\frac{F L}{P D}$ y por tanto $\frac{F L}{K L}>\frac{F L}{P D}$, luego $K L<P D=I L$. Así queda probado (2.11)

Interpretación del Teorema de Barrow:

Vamos a ver que el teorema de Barrow conduce a la relación $(\cdot)$, que es la forma de visualizar la conexión existente entre la derivada y la integral.

En efecto, sea $y=f(x)$ una función creciente y orientada hacia abajo; ahora se construye la curva $Y=$ $F(x)$ la que satisface la condición: "la ordenada genérica $Y=D F$ (ver anterior figura) representa el área determinada por la curva $y=f(x)$ y las ordenadas $F(0)=V Z$ y $f(x)=D E$ ”. Así, se asume $Y=\int_{0}^{x} f(t) d t$

Ahora, para construir la tangente a la curva $Y=F(x)$ en el punto $F(x, Y)$, Barrow partiendo del punto $D=(x, 0)$, coge sobre el eje $\mathrm{x}$ el punto $\mathrm{T}$ tal que tenga la relación $D T=\frac{Y}{y}=\frac{D F}{D E}$, luego, prueba que la recta $T F$ es tangente.

Por otro lado, se tiene $y=D E=\frac{D F}{D T}$ y el resultado es equivalente a $y=\frac{d Y}{d x}$, esto es, $y=\frac{d}{d x} \int_{0}^{x} f(t) d t$, ó $\frac{d}{d_{x}} \int_{0}^{x} f(t) d t=f(x)$.

3. Isaac Newton (1642-1727). Estamos alrededor del siglo XVII, época en que las ideas investigadas desde tiempos de los antiguos sobre la construcción de tangentes y cálculo de áreas van alcanzando su madurez. El surgimiento de dos genios, Newton y Leibniz, habría de culminar una etapa con la formulación de una bella y profunda teoría, el cálculo infinitesimal; y el inicio de otra, el análisis matemático. Newton basó mucho de su trabajo en las series para representar funciones y obtener nuevos resultados. Veamos.

\section{Ejemplo 3.1 (Binomio Generalizado de Newton (1,676)).}

El teorema del binomio, tal como es presentado a continuación, fue enviado vía una carta a G. W. Leibniz. $y$ dice (ver [2] y [7] para mayores detalles). "sean $m$ y $n$ dos enteros, entonces

$$
(P+P Q)^{\frac{m}{n}}=P^{\frac{m}{n}}+\frac{m}{n} A Q+\frac{m-n}{2 n} B Q+\frac{m-2 n}{3 n} C Q+\frac{m-3 n}{4 n} D Q+\ldots
$$

donde $P+P Q$ es el binomio considerado y los coeficientes son determinados vías las expresiones: $A=$ $P \frac{m}{n}, B=\frac{m}{n} A Q$

$$
\begin{gathered}
C=\frac{m-n}{2 n} B Q=\frac{(m-n) m}{(2 n) n} P^{\frac{m}{n}} Q^{2}=\frac{\left(\frac{m}{n}\right)\left(\frac{m}{n}-1\right)}{2} P^{\frac{m}{n}} Q^{2} \\
D=\frac{m-2 n}{3 n} C Q=\frac{\left(\frac{m}{n}\right)\left(\frac{m}{n}-1\right)\left(\frac{m}{n}-2\right)}{3 \times 2} P^{\frac{m}{n}} Q^{3}, \ldots
\end{gathered}
$$

Considerando estos valores de los coeficientes $A, B, C, \ldots$, factorizando $P \frac{m}{n}$ en ambos lados y cancelando se obtiene:

$$
(1+Q)^{\frac{m}{n}}=1+\frac{m}{n} Q+\frac{\left(\frac{m}{n}\right)\left(\frac{m}{n}-1\right)}{2} Q^{2}+\frac{\left(\frac{m}{n}\right)\left(\frac{m}{n}-1\right)\left(\frac{m}{n}-2\right)}{3 \times 2} Q^{3}+
$$

Esta expresión es más manejable para obtener expansiones interesantes, así como para observar como Newton llega a ciertas representaciones en series de algunas funciones.

Ejemplo 3.2. Expandir:

(i) $(1+x)^{3}$;

(ii) $(1+x)^{-3}$.

\section{Solución:}


(i) Usamos (3.1) con $Q=x, \frac{m}{n}=3$. De esta manera,

$$
(1+x)^{3}=1+3 x+\frac{3 \cdot 2}{2} x^{2}+\frac{3 \cdot 2 \cdot 1}{3 \cdot 2} x^{3}+\frac{3 \cdot 2 \cdot 1 \cdot 0}{4 \cdot 3 \cdot 2} x^{4}+\ldots=1+3 x+3 x^{2}+x^{3} .
$$

(ii) $(1+x)^{-3}=1+(-3) x+\frac{(-3)(-4)}{2} x^{2}+\frac{(-3)(-4)(-5)}{6} x^{3}+\ldots$, de donde

$$
\begin{aligned}
& \frac{1}{(1+x)^{3}}=1-3 x+6 x^{2}-10 x^{3}+15 x^{4}-\ldots \text { ó aún } \\
& \frac{1}{1+3 x+3 x^{2}+x^{3}}=1-3 x+6 x^{2}-10 x^{3}+15 x^{4}-\ldots
\end{aligned}
$$

Newton verificó que $\left(1+3 x+3 x^{2}+x^{3}\right)\left(1-3 x+6 x^{2}-10 x^{3}+15 x^{4}-\cdots\right)=1$.

Ejemplo 3.3. Expandir

(i) $\sqrt{1-x}$;

(ii) . Obtener una aproximación decimal de $\sqrt{7}$.

\section{Solución:}

(i) Tenemos $\sqrt{1-x}=(1-x)^{\frac{1}{2}}, Q=-x, \frac{m}{n}=\frac{1}{2}$, entonces

$$
\begin{aligned}
& \sqrt{1-x}=1+\frac{1}{2}(-x)+\frac{\left(\frac{1}{2}\right)\left(-\frac{1}{2}\right)}{2}(-x)^{2}+\frac{\left(\frac{1}{2}\right)\left(-\frac{1}{2}\right)\left(-\frac{3}{2}\right)}{6}(-x)^{3} \ldots \\
& =1-\frac{1}{2} x-\frac{1}{8} x^{2}-\frac{1}{16} x^{3}-\frac{5}{128} x^{4}-\frac{7}{256} x^{5}-\ldots \text { (+) }
\end{aligned}
$$

Newton verificó que:

$$
\begin{aligned}
& \left(1-\frac{1}{2} x-\frac{1}{8} x^{2}-\frac{1}{16} x^{3}-\frac{5}{128} x^{4}-\cdots\right)\left(1-\frac{1}{2} x-\frac{1}{8} x^{2}-\frac{1}{16} x^{3}-\frac{5}{128} x^{4}-\cdots\right) \\
& =1-x+0 x^{2}+0 x^{3}+0 x^{4}+\ldots=1-x .
\end{aligned}
$$

(ii) Tenemos $7=9\left(\frac{7}{9}\right)=9\left(1-\frac{2}{9}\right)$; luego $\sqrt{7}=3 \sqrt{1-\frac{2}{9}}$, y por (3.1)

$$
\sqrt{7} \simeq 3\left(1-\frac{1}{9}-\frac{1}{162}-\frac{1}{1458}-\frac{5}{52488}-\frac{7}{472392}\right)=2,64576 \ldots
$$

Ejemplo 3.4 (Aproximación de $\pi$ según Newton).

Sea el semicírculo ADE con centro $C=\left(\frac{1}{2}, 0\right)$, con radio $\frac{1}{2}$. Newton sabía que $\left(x-\frac{1}{2}\right)^{2}+(y-0)^{2}=$ $\left(\frac{1}{2}\right)^{2}$, de donde

$$
\begin{aligned}
& x^{2}-x+\frac{1}{4}+y^{2}=\frac{1}{4} \text { ó } y=\sqrt{x-x^{2}}=x^{\frac{1}{2}}(1-x)^{\frac{1}{2}}= \\
& x^{\frac{1}{2}}\left(1-\frac{1}{2} x-\frac{1}{8} x^{2}-\frac{1}{16} x^{3}-\frac{5}{128} x^{4}-\frac{7}{256} x^{5}-\cdots\right) \\
& =x^{\frac{1}{2}}-\frac{1}{2} x^{\frac{3}{2}}-\frac{1}{8} x^{\frac{5}{2}}-\frac{1}{16} x^{\frac{7}{2}}-\frac{5}{128} x^{\frac{9}{2}}-\frac{7}{256} x^{\frac{11}{2}}-\ldots
\end{aligned}
$$

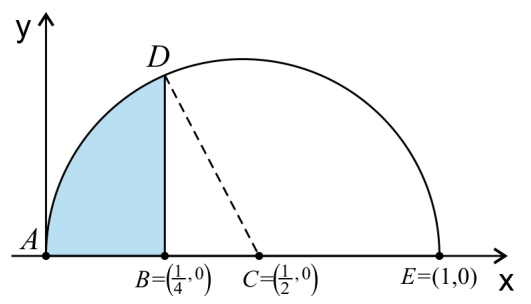

Figura 3.1: Aproximación de $\pi$ segun Newton

Ahora aparece el genio de Newton: elige $B=\left(\frac{1}{4}, 0\right)$ y traza $\overline{B D} \perp \overline{A E}$ y ataca el área sombreada $A B D$ en dos formas diferentes:

(i). Área de $A B D$ vía el método de las fluxiones. 


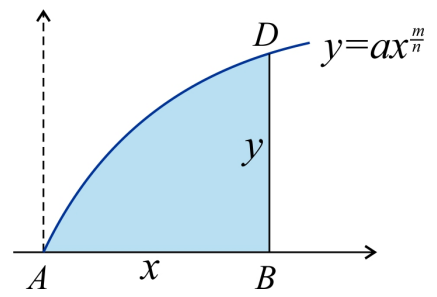

Figura 3.2: Método de Fluxiones para calcular áreas

Newton conocía como calcular el área bajo una curva; veamos la idea.

Sea la base $\overline{A B}$ de cualquier curva $A D$ que tiene $\overline{B D}$ como su perpendicular ordenada; llamemos $A B=$ $x, B D=y$, y sean $a, b, c$ etc, cantidades dadas $m, n$ números enteros.

Entonces se tiene:

Regla 1. Si $a x^{\frac{m}{n}}=y$ entonces área de $A B D=\frac{a n}{m+n} x^{\frac{m+n}{n}}$.

Regla 2. Si el valor de y tiene varios términos, el área sabiamente es la suma de las áreas que resulta de los términos.

Por ejemplo, si $y=x, a=m=n=1$, la regla 1 dice el respectivo triángulo rectángulo tiene área $\frac{1}{2} x^{2}$. Por otro lado, por la regla 2, el área bajo la curva $y=x^{2}+x^{\frac{3}{2}}$ es igual a $\frac{1}{3} x^{3}+\frac{2}{5} x^{\frac{5}{2}}$.

Volvamos a nuestro problema (i). Aplicando estas reglas, el área de la región sombreada en la semicircunferencia es:

$$
\begin{aligned}
& A=\frac{2}{3} x^{\frac{3}{2}}-\frac{1}{2}\left(\frac{2}{5} x^{\frac{5}{2}}\right)-\frac{1}{8}\left(\frac{2}{7} x^{\frac{7}{2}}\right)-\frac{1}{16}\left(\frac{2}{9} x^{\frac{9}{2}}\right)-\ldots \\
& =\frac{2}{3} x^{\frac{3}{2}}-\frac{1}{5} x^{\frac{5}{2}}-\frac{1}{28} x^{\frac{7}{2}}-\frac{1}{72} x^{\frac{9}{2}}-\frac{5}{704} x^{\frac{11}{2}}-\ldots
\end{aligned}
$$

Esta representación Newton la evalúa en $x=\frac{1}{4}$ y se observa que $\left(\frac{1}{4}\right)^{\frac{3}{2}}=\frac{1}{8}$,

$\left(\frac{1}{4}\right)^{\frac{5}{2}}=\frac{1}{32},\left(\frac{1}{4}\right)^{\frac{7}{2}}=\frac{1}{128}, \ldots ;$ de esta manera, el área aproximada de $A B D$ considerando los nueve primeros términos es ( en $\left.x=\frac{1}{4}\right)$ :

$$
A \approx \frac{1}{12}-\frac{1}{160}-\frac{1}{3584}-\frac{1}{36864}-\frac{5}{1441792}-\ldots-\frac{429}{163208757248} \approx 0,07677310678
$$

Curiosidad: Hoy en día cálculos como el presente se hacen rápidos con la ayuda de buenas calculadoras; ¿Cómo se hicieron en la época de Newton?...

(ii). El cálculo del área de $A B D$ vía la geometría.

Newton re-examina el problema del área sombreada $A B D$ vía argumentos geométricos. Observa que $B D=\sqrt{\left(\frac{1}{2}\right)^{2}-\left(\frac{1}{4}\right)^{2}}=\frac{\sqrt{3}}{4}$; luego área del triángulo $D B C=\frac{1}{2}\left(\frac{1}{4}\right)\left(\frac{\sqrt{3}}{4}\right)=\frac{\sqrt{3}}{32}$.

Ahora Newton determina el área del sector $A C D$. Observa que en el triángulo $D B C$, el ángulo $B C D$ mide $60^{\circ}$ y que: área el sector $A C D=\frac{1}{3}$ área del semicírculo $=\frac{1}{3}\left(\frac{1}{2} \pi r^{2}\right)=\frac{\pi}{24}$.

Luego, Área $(A B D)=$ área del sector $A C D$ - área del triángulo $D B C$ $=\frac{\pi}{24}-\frac{\sqrt{3}}{32}$.

Es decir, Newton llega al área de ABD vía dos caminos, y por tanto se tiene:

$0,07677310678 \approx \frac{\pi}{24}-\frac{\sqrt{3}}{32}$,

de donde se obtiene un valor aproximado de $\pi$ (que es el objetivo):

$$
\pi \approx 24\left(0,07677310678+\frac{\sqrt{3}}{32}\right)=3,141592668
$$

Nota. Remarcamos que para Newton el cálculo es un álgebra de series infinitas ! y establece su punto de vista sobre el rol de las series de potencias; así en su estudio sobre la cuadratura de la hipérbola, Newton llega a establecer (1668):

$$
\int_{0}^{x} \frac{1}{1+t} d t=x-\frac{x^{2}}{2}+\frac{x^{3}}{3}-\frac{x^{4}}{4}+\ldots
$$

Observación 3.1. Se sabe que los hindúes conocieron, de algún modo, las series asociadas a las funciones $t^{-1} x$, senx, $\cos x$, series que fueron re-descubiertas por Newton con su método. Además, estableció la 
Regla 3. "En orden a calcular el área bajo la curva $f(x, y)=0$, uno debe expandir " $y$ " como una suma de términos de la forma ax ${ }^{\frac{m}{n}}$ y luego se aplican las reglas 1 y 2 ”.

\section{Ejemplo 3.5 (Las Series y el Cálculo Infinitesimal.).}

El camino usado por Newton para crear el cálculo infinitesimal, vía su método de fluxiones estuvo fuertemente ligado a las series; en efecto, usando series Newton pudo estudiar funciones algebraicas básicas así como algunas funciones trascendentales. La idea era desarrollar la función en serie y luego las derivaba o las integraba término a término; por otro lado, Leibniz también usó las series en su teoría pero sin la fuerza que le dio Newton. Sin embargo, las series tomaron un gran impulso en el siglo XVIII y a partir de entonces forma una parte importante del cálculo infinitesimal; en este siglo existieron grandes matemáticos donde destaca nítidamente el gran matemático Euler, como debemos contribuciones a Lagrange y a la familia Bernoulli. Si bien la idea de serie surge en la antigüedad (Arquímedes), en el siglo XVIII Mercator $y$ Newton descubrieron la serie $\log (1+x)=x-\frac{1}{2} x^{2}+\frac{x^{3}}{3}-\frac{x^{4}}{4}+\ldots$,

Observando que para $x=2$ la serie es infinita en tanto que a la derecha se tiene log3 (un valor finito). Estas "rarezas" se debieron a que en esa época no se tenía la idea de "dominio de convergencia" de una serie (en este caso la serie converge para $-1<x<1$ ). Tal rareza fue observada por Wallis pero no tuvo una explicación al respecto; en esta dirección James Gregory (1638-1675) usando un método de Wallis obtuvo la serie $x=\operatorname{tg} x-\frac{1}{3} \operatorname{tg}^{3} x+\frac{1}{5} \operatorname{tg}^{5} x-\ldots$, de donde si $x=\frac{\pi}{4}$ se obtiene $\frac{\pi}{4}=1-\frac{1}{3}+\frac{1}{5}-\frac{1}{7}+\ldots y$ así se podría obtener un valor aproximado para $\pi$.

Gregory fue uno de los primeros matemáticos en distinguir entre una serie convergente y una divergente; también anunció en 1671 que había obtenido, entre otras, las series

$\operatorname{tg} x=x+\frac{x^{3}}{3}+\frac{2}{15} x^{5}+\frac{17}{317} x^{7}+\ldots$

$\sec x=1+\frac{1}{2} x^{2}+\frac{5}{24} x^{4}+\frac{61}{720} x^{6}+\ldots$

en donde se remarca que no se conoce como Gregory consiguió estas series. En esta dirección, Leibniz. obtuvo las series para senx, cosx y arctgx, y Newton obtuvo la serie geométrica

$\frac{1}{1-x}=1+x+x^{2}+x^{3}+\ldots(*)$, integrando esta serie obtuvo la serie del logaritmo

$\log (1+x)=x-\frac{x^{2}}{2}+\frac{x^{3}}{3}-\frac{x^{4}}{4}+\ldots$, como enunciamos anteriormente.

Siguiendo su método, Newton de la serie $\frac{1}{1+x^{2}}=1-x^{2}+x^{4}-x^{6}+\ldots$ obtuvo la siguiente representación $\operatorname{arctg} x=x-\frac{x^{3}}{3}+\frac{x^{5}}{5}-\frac{x^{7}}{7}+\ldots$. Por otro lado, las series fueron útiles para calcular el valor de números especiales como $\pi$, e, así como representar funciones trigonométricas y logarítmicas; Newton, Leibniz, Gregory, entre otros, tuvieron interés en las series con este objetivo. Así, Leibniz en 1674 obtuvo la representación $\frac{\pi}{4}=1-\frac{1}{3}+\frac{1}{5}-\frac{1}{7}+\ldots$ obtenida también por Gregory como caso particular del desarrollo de $f(x)=x$; recordemos que Arquímedes también hizo esfuerzos por calcular una buena aproximación para $\pi$.

\section{Ejemplo 3.6 (Newton).}

De la anterior representación (*) para $\frac{1}{1-x}$, poniendo $-x$ por $x$ se obtiene $\frac{1}{1+x}=1-x+x^{2}-x^{3}+x^{4}-$ ..., la misma que se puede obtener haciendo una división directa de 1 por $1+x$. Veamos:

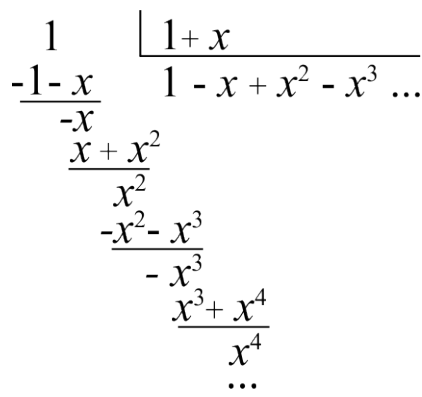

Históricamente la representación (*) fue usada por Arquímedes y Fermat para el cálculo de áreas y por Newton en su teorema del binomio, quien usó la regla 3 en su estudio de la hipérbola $y=\frac{a^{2}}{b+x}$. Veamos como Newton obtuvo una representación en series para $\frac{a^{2}}{b+x}$. 


$$
\begin{aligned}
& \frac{a^{2}-\frac{a^{2}}{b} x}{-\frac{a^{2}}{b} x} \quad \frac{b+x}{\frac{a^{2}}{b}-\frac{a^{2}}{b^{2}} x+\frac{a^{2}}{b^{3}} x^{2}-\frac{a^{2}}{b^{4}} x^{3}+\cdots} \\
& \frac{\frac{a^{2}}{b} x+\frac{a^{2}}{b^{2}} x^{2}}{\frac{a^{2}}{b^{2}} x^{2}} \\
& -\frac{a^{2}}{b^{2}} x^{2}-\frac{a^{2}}{b^{3}} x^{3} \\
& -\frac{a^{2}}{b^{3}} x^{3}
\end{aligned}
$$

Por lo tanto, $\frac{a^{2}}{b+x}=\frac{a^{2}}{b}-\frac{a^{2} x}{b^{2}}+\frac{a^{2} x^{2}}{b^{3}}-\frac{a^{2} x^{3}}{b^{4}}+\ldots$.

$Y$ de esta manera, por la regla 3, el área bajo la hipérbola es dada por (integrando término a término !) $A=\frac{a^{2}}{b} x-\frac{a^{2}}{2 b^{2}} x^{2}+\frac{a^{2}}{3 b^{3}} x^{3}-\frac{a^{2}}{4 b^{4}} x^{4}+\ldots$

En forma similar, vía una división directa, Newton observó que

$\frac{1}{1+x^{2}}=1-x^{2}+x^{4}-x^{6}+x^{8}-\ldots$, y por tanto obtuvo Newton que el área bajo el gráfico de $y=\frac{1}{1+x^{2}}$, del origen al punto $x$, es: $A=x-\frac{1}{3} x^{3}+\frac{1}{5} x^{5}-\frac{1}{7} x^{7}+\frac{1}{9} x^{9}-\ldots$.

Pero, por otro lado, se sabe que $\int \frac{1}{1+x^{2}} d x=\operatorname{arctg} x+C$, donde $C=0$ desde que el área es cero si $x=0$. Luego, arctg $x=x-\frac{1}{3} x^{3}+\frac{1}{5} x^{5}-\frac{1}{7} x^{7}+\frac{1}{9} x^{9}-\ldots$, como ya habíamos mencionado anteriormente, serie que también conocía Gregory. Si $x=1$, arctg $1=\frac{\pi}{4}$ y se obtiene la representación de Leibniz, también mencionada anteriormente.

\section{Ejemplo 3.7 (Newton.- Ecuaciones Diferenciales).}

Newton fue un genial físico-matemático y como tal estuvo interesado en interpretar los fenómenos físicosastronómicos de su época; en esta dirección su cálculo infinitesimal fue aplicado exitosamente para resolver diversos problemas matemáticos y científicos en general los que son reducidos a resolver ecuaciones diferenciales. Así, en su trabajo "Sobre el Método de Fluxiones ..." aparecen investigaciones en este sentido en donde Newton introduce diferentes técnicos para resolver tales ecuaciones. La idea fue reemplazar la función " $y$ ” y sus derivadas por series de potencias con coeficientes desconocidos los que son determinados usando la ecuación dada. Bien, en lo que sigue daremos atención a la primera derivada y' de y; es claro que los ejemplos a ser presentados son puestos en el contexto de la época de Newton y no tienen el rigor que ahora conocemos.

Sea la ecuación diferencial $y^{\prime}=x+y$. La idea es representar a la solución de esta ecuación con una serie infinita del tipo

$$
y=c_{o}+c_{1} x+c_{2} x^{2}+c_{3} x^{3}+c_{4} x^{4}+\ldots=\sum_{n=0}^{\infty} c_{n} x^{n} .
$$

Derivando, término a término, se obtiene

$$
\begin{aligned}
& y^{\prime}=c_{1}+2 c_{2} x+3 c_{3} x^{2}+4 c_{4} x^{3}+\ldots=\sum_{n=1}^{\infty} \sum n c_{n} x^{n-1} . \\
& =\sum_{n=0}^{\infty}(n+1) c_{n+1} x^{n} .
\end{aligned}
$$

Estas representaciones son llevadas a la ecuación original y se obtiene $\sum_{n=0}^{\infty}(n+1) c_{n+1} x^{n}=x+\sum_{n=0}^{\infty} c_{n} x^{n}$. Ahora como Newton hizo, se identifican los coeficientes $c_{n}$ de ambos lados de esta ecuación para $n=$ $0,1,2, \ldots$; así, si $n=0$, se tiene $c_{1}=c_{0}$; si $n=1,2 c_{2}=1+c_{1}$ ó $c_{2}=\frac{1+c_{1}}{2}$;

Si $n=2, \quad 3 c_{3}=c_{2}$ ó $c_{3}=\frac{c_{2}}{3}=\frac{1+c_{1}}{2 \cdot 3}$,

Si $n=3,4 c_{4}=c_{3}$ ó $c_{4}=\frac{c_{3}}{4}=\frac{1 .+c_{1}}{2 \cdot 3 \cdot 4}$,

Si $n=4,5 c_{5}=c_{4}$ ó $c_{5}=\frac{c_{4}}{5}=\frac{1+c_{1}}{2 \cdot 3 \cdot 4 \cdot 5}$, 
En general para $n \geq 2, c_{n}=\frac{1+c_{1}}{n !}$.

Si ponemos $c=c_{0}$, entonces

$c_{1}=c, c_{2}=\frac{1+c}{2}, c_{3}=\frac{1+c}{2 \cdot 3}, \ldots c_{n}=\frac{c_{n-1}}{n}=\frac{1+c}{n !}$.

De esta manera, la solución general de la ecuación $y^{\prime}=x+y$ es la función

$$
y=c+c x+(1+c)\left(\frac{x^{2}}{2 !}+\frac{x^{3}}{3 !}+\frac{x^{4}}{4 !}+\frac{x^{5}}{5 !}+\ldots\right) .
$$

Usando el método de las series infinitas resolver la ecuación diferencial $y^{\prime}=y+\frac{1}{1-x}$.

\section{Solución:}

Tenemos $\sum_{n=0}^{\infty}(n+1) c_{n+1} x^{n}=\sum_{n=0}^{\infty} c_{n} x^{n}+1+x+x^{2}+x^{3}+\ldots$.

Luego, si $n=0, c_{1}=c_{0}+1 ; n=1,2 c_{2}=c_{1}+1$, de ello $c_{2}=\frac{C_{1+1}}{2}=\frac{c_{0}+2}{2}$;

si $n=2,3 c_{3}=c_{2}+1$; luego $c_{3}=\frac{c_{2+1}}{3}=\frac{c_{0}+4}{2 \cdot 3}$;

si $n=3,4 c_{4}=c_{3}+1$; luego $c_{4}=\frac{c_{3+1}}{4}=\frac{c_{0}+10}{2 \cdot 3 \cdot 4} ; \ldots$

Luego, si $c_{0}=c$, la solución de la ecuación $y^{\prime}=y+\frac{1}{1-x}$ es la función

$y=c_{o}+c_{1} x+c_{2} x^{2}+c_{3} x^{3}+\ldots=c+(1+c) x+\left(\frac{2+c}{2}\right) x^{2}+\left(\frac{4+c}{6}\right) x^{3}+\left(\frac{10+c}{24}\right) x^{4}+\ldots$

\section{Gottfried W. Leibniz (1646-1716).}

"Leibniz es uno de los más poderosos espíritus de la civilización occidental".

Enciclopedia Británica.

El cálculo infinitesimal fue la culminación de todo un proceso histórico de muchos años y llegó a formalizarse (no en forma muy consistente) con la mente de dos genios excepcionales, I. Newton y G. G. Leibniz. A partir de este momento se inicia una nueva aventura matemática la que culminaría, de alguna manera, con la formulación del análisis matemático en el siglo XIX. Tanto Leibniz, como Newton, dejaron muchos escritos que solo se conocieron muchos años después; como sabemos Newton no gustaba publicar sus trabajos, y los que se publicación fue por presión de sus íntimos amigos; fueron sus descendientes quienes dieron a conocer sus restantes escritos. Así mismo, mucho de la obra de Leibniz también fue inédita y sus escritos y su correspondencia se dio a conocer a inicios del siglo XX surgiendo el proyecto de editar todos sus escritos, algo que parece no termina aun pues son alrededor de veinte mil cartas (menos mal que Leibniz tuvo la costumbre de no destruir sus papeles). Leibniz tuvo una mente universal pues cultivó con profundidad el derecho, la religión, la historia, la política, la lógica, la metafísica, la botánica, la filosofía y desde luego la matemática, es decir, fue un polifacético por excelencia; al aspecto que nos interesa resaltar es su interés por la matemática, en particular, por su contribución creando el cálculo infinitesimal; si solo hubiera hecho esta investigación habría también pasado a la historia de la matemática pero hizo muchísimo más en otras áreas. Leibniz fue contemporáneo con Newton y llegaron a conocerse; mientras Newton fue un filósofo natural que investigó modelos matemáticos para interpretar al universo físico, Leibniz fue un profundo filósofo que buscaba un lenguaje universal para explicar a la naturaleza; fue un pensador por excelencia y tuvo una altísima cultura de casi todo el conocimiento de su época.

\section{Ejemplo 4.1 (Leibniz - 1672.- Huygens planteó a Leibniz el siguiente).}

Problema. "Sean los números triangulares 1, 3, 6, 10, 15, ... y sus sucesivas diferencias 2, 3, 4, 5, números que son dados en general vía $\frac{i(i+1)}{2}, i=1,2,3, \ldots$ Calcular

$$
\frac{1}{1}+\frac{1}{3}+\frac{1}{6}+\frac{1}{10}+\ldots+\frac{2}{n(n+1)}+\ldots
$$

Solución: Leibniz tuvo la genialidad de hacer la siguiente descomposición (hoy día usada):

Luego (4.1) puede ser escrito en la forma

$$
\frac{2}{n(n+1)}=2\left(\frac{1}{n}-\frac{1}{n+1}\right)
$$

$$
\left(\frac{2}{1}-\frac{2}{2}\right)+\left(\frac{2}{2}-\frac{2}{3}\right)+\left(\frac{2}{3}-\frac{2}{4}\right)+\left(\frac{2}{4}-\frac{2}{5}\right)+\ldots+\left(\frac{2}{n}-\frac{2}{n+1}\right)+\ldots
$$

lo que lleva a $2-\frac{2}{n+1}$, y conforme $n$ crece, y crece, esto conduce a 2 . $\square$ 
De un modo más general, desde que $\frac{2}{i(i+1)}$ es reconocido como una sucesión diferencia de los términos en nuevo modelo $\left(\frac{2}{i}\right)$, la n-ésima suma parcial de la serie original puede ser computada como la diferencia entre el primer término y el n-termino en el nuevo modelo. Esta observación nos expresa la esencia del "teorema fundamental del cálculo". Leibniz estudia este fenómeno posteriormente en el llamado triángulo armónico. Veamos este "triángulo" y el aritmético.

$\begin{array}{cccccccc}1 & 1 & 1 & 1 & 1 & 1 & 1 & \ldots \\ 1 & 2 & 3 & 4 & 5 & 6 & \ldots & \\ 1 & 3 & 6 & 10 & 15 & \ldots & & \\ 1 & 4 & 10 & 20 & \ldots & & & \\ 1 & 5 & 15 & \ldots & & & & \\ 1 & 6 & \ldots & & & & & \\ 1 & \ldots & & & & & & \end{array}$

Triángulo Aritmético

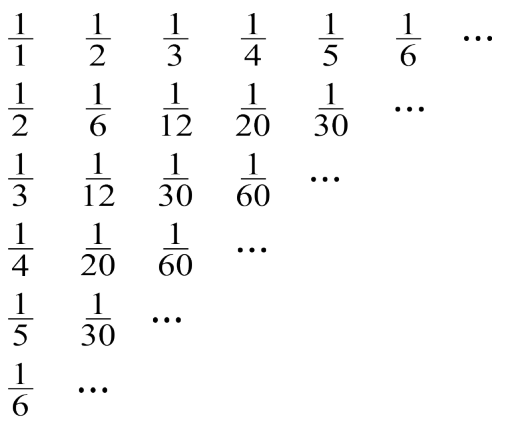

Triángulo Armónico

Leibniz hizo la siguiente inteligente observación: "formando sucesiones diferencias y sumas de sucesiones, ellas son mutuamente operaciones inversas".

Análogamente piensa en el problema del área como una suma de diferencias infinitesimales, lo que lo conduce a la conexión entre área y tangente! (Teorema-Fundamental del cálculo infinitesimal).

Ejemplo 4.2 (Idea de Leibniz para Llegar al Cálculo Infinitesimal).

¿Cuál fue la idea fundamental para que Leibniz llegara a su cálculo? Veamos. Tanto Pascal como Barrow utilizaron como instrumento de trabajo al triángulo característico (ver posterior figura (•)) pero con objetivos diferentes pues para Pascal fue un instrumento para resolver problemas de cuadraturas mientras que Barrow lo utilizó para calcular la tangente a una curva. En tanto, Leibniz tuvo el genio de conjeturar que entre ambos problemas existe una relación recíproca, pero, además observó su utilidad en el estudio de las curvas arbitrarias y así su idea básica para entender su nuevo método fue "considerar una curva como un polígono de infinitos lados cuyas longitudes son infinitamente pequeñas. Veamos gráficamente esta idea.

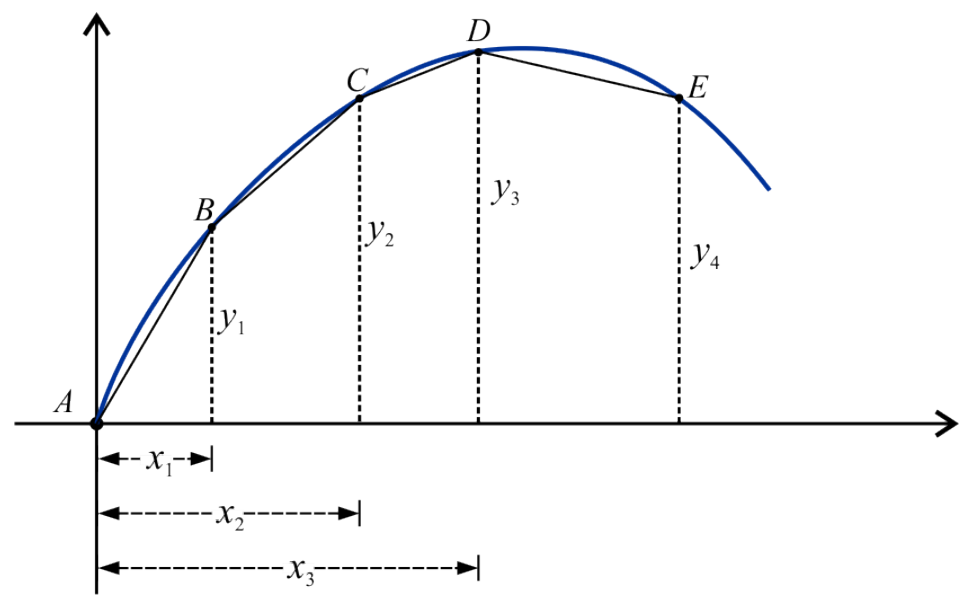

Figura 4.1: Idea de Leibniz sobre el cálculo

En el siguiente argumento apreciamos la idea esencial de como Leibniz llega a los dos problemas básicos del cálculo infinitesimal. Para ello sean $A, B, C, D, \ldots$ los vértices de un polígono inscrito en la curva dada, polígono que es una aproximación "tosca" de la curva.

De esta manera se tienen dos sucesiones de variables: las abscisas $x_{1}, x_{2}, x_{3}, \ldots$ y las ordenadas $y_{1}, y_{2}, y_{3}$, ... de los vértices. Ahora la idea es escoger las abscisas de modo que $x_{i+1}-x_{i}=1$; entonces $y \cdot 1$ es el área del rectángulo respectivo y de esta manera $y_{1}+y_{2}+y_{3}+\ldots$ nos da una aproximación del área bajo la curva, esto es la cuadratura. 
Por otra parte, la diferencia $y_{i+1}-y_{i}=\frac{y_{i+1}-y_{i}}{x_{i+1}-x_{i}}$ es una aproximación de la pendiente de la tangente en el punto correspondiente de la curva. Visualmente y geométricamente observamos que estas aproximaciones se mejoran conforme las diferencias entre las abscisas consecutivas se hacen cada vez más y más pequeñas, $y$ de esta manera se resuelven el problema de la tangente y el de la cuadratura cuando se considera que el polígono tiene infinitos lados, y estos con dimensiones infinitamente pequeños. Como observamos, Leibniz fue un matemático con una intuición e imaginación genial no obstante que él no se entregó por completo a la matemática y fue también grande en diversas otras áreas. Pero, sigamos con estos argumentos.

Veamos nuevamente a la anterior figura. Leibniz observa que si en la sucesión $y_{1}, y_{2}, y_{3}$, .. se considera las sumas $y_{1}, y_{1}+y_{2}, y_{1}+y_{2}+y_{3}, \ldots$ se observa que $\left(y_{1}+y_{2}\right)-y_{1}=y_{2},\left(y_{1}+y_{2}+y_{3}\right)-\left(y_{1}+y_{2}\right)=y_{3}, \ldots$ es decir se obtiene la sucesión dada.

Recíprocamente, con la sucesión dada $y_{1}, y_{2}, y_{3}, \ldots$ se forman la sucesión diferencias $y_{1}, y_{2}-y_{1}, y_{3}-$ $y_{2}, \ldots$; ahora se observa que $\left(y_{2}-y_{1}\right)+y_{1}=y_{2},\left(y_{3}-y_{2}\right)+\left(y_{2}-y_{1}\right)+y_{1}=y_{3}, \ldots$ y se vuelve a obtener la sucesión dada.

Por estos argumentos Leibniz concluye que los problemas de la cuadratura de una curva y el hallazgo de su tangente son problemas, uno el inverso del otro, y en esto descansa el poderío del cálculo infinitesimal.

Por otro lado, Leibniz estuvo muy preocupado por encontrar un lenguaje universal y notaciones matemáticas que sean sencillas y operativas. En esta dirección 1684 es histórico pues en este año se publica por primera vez un trabajo sobre el cálculo diferencial; así, en la revista Acta Eruditorum hay un artículo de Leibniz en donde expone las ideas básicas de su teoría siendo su título muy sugestivo:

“ Un nuevo método para encontrar máximos y mínimos así como tangentes, para el cual no son obstáculos ni las cantidades fraccionarias ni las irracionales, y un notable tipo de cálculo para esto ”.

Ejemplo 4.3 (Nosotros Usamos la Notación de Leibniz).

En el "Nuevo Método ... " Leibniz considera notaciones que ahora usamos frecuentemente; así en el plano $d x$ e dy son incrementos pequeños finitos; $\frac{d y}{d x}$ representa la derivada de y con respecto a $x$. Así, si $y=x^{2}$ $y \Delta x$ es un pequeño cambio en $x$, ¿cómo es el cambio $\Delta y$ ? ... Se tiene (en lenguaje actual)

$\Delta y=(x+\Delta x)^{2}-x^{2}=x^{2}+2 x \Delta x+(\Delta x)^{2}-x^{2}=2 x \Delta x+(\Delta x)^{2}$,

$y$ la razón de cambio es $\frac{\Delta y}{\Delta x}=2 x+\Delta x$. Ahora, la idea es hacer $\Delta x$ tan pequeño como se quiera (en valor absoluto), (es decir, $\Delta x \rightarrow 0$ ) para obtener

$\lim _{\Delta x \rightarrow 0} \frac{\Delta y}{\Delta x}=\lim _{\Delta x \rightarrow 0}(2 x+\Delta x)=2 x$.

Por definición este límite, $2 x$, es la derivada de $x^{2}$ con respecto a $x$.

Leibniz considera la notación $\frac{d y}{d x}$ para expresar el $\lim _{\Delta x \rightarrow 0} \frac{\Delta y}{\Delta x}$.

Luego, $\frac{d y}{d x}=2 x$.

Llega establecer que $d(x y)=x d y+y d x ; d\left(\frac{x}{y}\right)=\frac{y d x-x d y}{y^{2}} ; d\left(x^{n}\right)=n x^{n-1}$.

Es anecdótico que recordamos más a Newton como el creador del cálculo infinitesimal que a Leibniz aun cuando la formulación de su teoría ha sido hecha con un lenguaje y estilo más claro que el de Newton. Posiblemente la balanza se inclinó por Newton por sus grandes aplicaciones que hizo del cálculo a la física y a la astronomía.

El "Nuevo Método ... " es considerado como el inicio del cálculo de las diferenciales; ahí Leibniz expone sus ideas fundamentales a ser desarrolladas posteriormente. Se discute el siguiente:

Problema (de De Beaune). En 1639 Florimont De Beaune propuso a Descartes la siguiente cuestión:

"Hallar una curva cuya subtangente sea una constante dada a.

Solución. Viendo la figura adjunta, tenemos que $\frac{d x}{d y}=\frac{s}{y}$

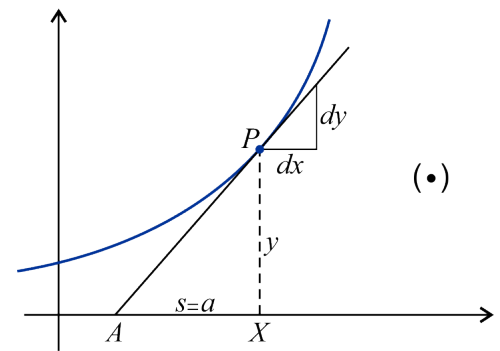

Figura 4.2: La curva logaritmica 
Si $s=a$, se obtiene ady $=y d x$. Pues bien, Leibniz considera $d x=b$ constante, es decir, esto equivale a tener las abscisas en progresión aritmética. Si $k=\frac{b}{a}$ se tendrá $d y=k y$, es decir, los incrementos son proporcionales a sus ordenadas. Veamos esto más explícitamente.

Sea la sucesión de abscisas $x_{0}=x, x_{1}=x+b, x_{2}=x+2 b, x_{3}=x+3 b$, ..., la que es una progresión aritmética; por otro lado, $d y_{1}=y_{1}-y_{0}=k y_{1}$, de donde $y_{1}=\frac{y_{0}}{1-k} \equiv k_{1} y_{0}$; de esta manera las correspondientes ordenadas $y_{0}, y_{1}=k_{1} y_{0}, y_{2}=k_{1}^{2} y_{0}, y_{3}=k_{1}^{3} y_{0}$, ... están en progresión geométrica.

Conclusión: Leibniz afirma que "la curva es una logarítmica"

Nota. En el lenguaje actual, Leibniz de la relación que define a la curva $a d y=y d x$ obtendría $a \frac{d y}{y}=d x$, de donde integrando se obtiene alny $=x+C$, una relación implícita.

Ejemplo 4.4. $\int x d y=x y-\int y d x . \quad \int x d x=\frac{x^{2}}{2}$,

Como observamos debemos a Leibniz diversos resultados, y notaciones, que actualmente usamos; es admirable que en tan poco tiempo (comenzó a estudiar matemática no muy joven) y con muchas otras ocupaciones hiciera grandes progresos como para crear una teoría tan profunda como es el cálculo infinitesimal. Leibniz fue un genio como también lo fue Newton.

Bien, las ideas de Leibniz sobre el cálculo fueron publicados desde 1684 pero muchas de sus ideas estuvieron en numerosos papeles hechos desde 1673, documentos que nunca publicó Leibniz.

Por otro lado, como comprenderemos, en la evolución de la teoría las notaciones eran complejas y no practicas e iban cambiando; así, en 1714 Leibniz escribió la obra "Historia et Origo Calculi Differentialis" en donde da un panorama de su teoría, de su punto de vista; este libro debe tener un gran valor histórico y en donde se siente la influencia de la polémica que tuvo con Newton. En relación a las notaciones introducidas por Leibniz veamos lo siguiente. Leibniz a la cantidad “ $d x$ ”, que frecuentemente la designa con " a”, es siempre $\ell$ (la diferencia de orden de dos términos sucesivos; la cantidad " dy" representa la primera diferencia de dos términos sucesivos. Por otro lado, con "omn" (significa "suma” en latín) Leibniz representa la suma de las primeras diferencias de una sucesión de términos que comienzan con cero; posteriormente Leibniz diría: "será útil escribir $\int$ en vez de omn, y de esta manera $\int \ell=$ omn $\ell$ o la suma de las $\ell$ ". Posteriormente introducirá la letra d para denotar diferencias. Después la “ $\ell$ ” reemplaza a "dy" y de esta manera Leibniz escribe omn $\cdot \ell=y$, donde y es el último de la sucesión. Así, vía su cálculo de diferencias, el área de un triángulo (rectángulo e isósceles) es: omn $\cdot y \ell=\frac{y^{2}}{2}$. Para comprender mejor esta conclusión veamos la siguiente figura.

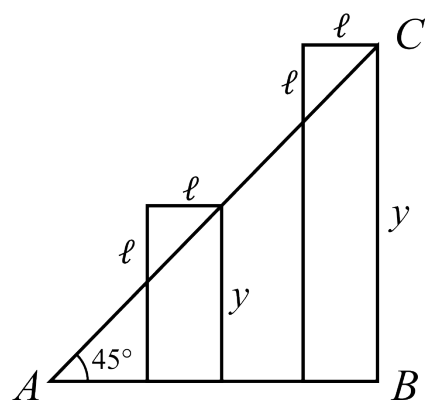

Figura 4.3: Leibniz y su cálculo de diferencias

Se considera la función $y=x$ (hoy sabemos que $\int_{o}^{x} t d t=\frac{x^{2}}{2}$ ), así el ángulo $B A C$ mide $45^{\circ}$. Según la idea de Leibniz el área del $\triangle B A C$ es la suma de los y $\ell$, con $\ell$ pequeño. El paso crucial fue el de ir del caso discreto al caso continuo; luego de tener dificultades diversas, Leibniz pasó al dx e dy de una función.

Así, en 1675 considera omn $\cdot y \ell=\overline{o m n} \cdot \overline{o m n \cdot \ell} \frac{\ell}{a}(+)$ donde está considerando $y=$ omn $\cdot \ell$ y donde divide $\ell$ por a para conservar la dimensión.

Leibniz afirma que (+) se verifica para todo $\ell . D e(+)$ y de omn $\cdot y \ell=\frac{y^{2}}{2}$ se obtiene omn $\cdot \overline{\text { omn } \cdot \ell} \frac{\ell}{a}=\frac{y^{2}}{2}$. ¿Cómo se interpreta esta igualdad a la luz actual? ... se observa que

$$
\int\left[\int d y\right] \frac{d y}{d x}=\int y \frac{d y}{d x}=\frac{y^{2}}{2} .
$$


El genio de Leibniz obtuvo otros interesantes resultados, como la integración por partes: omn $\cdot x \ell=$ $x o m n \cdot \ell-o m n \cdot o m n \ell(*)$ donde $\ell$ es la diferencia en valores de dos términos sucesivos de una sucesión, $x$ es el número del término. Leibniz, obtuvo (*) vía argumentos geométricos y actualmente es traducido como

$$
\int x d y=x y-\int y d x \text {. }
$$

Si ahora se hace $x=\ell$, como Leibniz hizo, se obtendrá de (*)

$$
\text { omn } \cdot x^{2}=x o m n \cdot x-\text { omn } \cdot \text { omn } \cdot x \text {, }
$$

pero Leibniz afirma que omn $\cdot x=\frac{x^{2}}{2}\left(\right.$ recordemos que omn $\left.\cdot y \ell=\frac{y^{2}}{2}\right)$, luego omn $\cdot x^{2}=x \frac{x^{2}}{2}-$ omn $\cdot \frac{x^{2}}{2}$, de donde omn $\cdot x^{2}+$ omn $\frac{x^{2}}{2}=\frac{x^{3}}{2}$ ó $\frac{3}{2}$ omn $\cdot x^{2}=\frac{x^{3}}{2}$, es decir omn $\cdot x^{2}=\frac{x^{3}}{3}$. Observemos bien esta igualdad.

Era el 29 de octubre de 1675 cuando Leibniz decide escribir $\int$ en vez de $o m n \cdot$, y de esta manera $\int \ell=$ omn $\cdot \ell$, así como $\int x=\frac{x^{2}}{2}, \int x^{2}=\frac{x^{3}}{3}, \ldots$

(Ver [1], [8] y [7] para otros detalles).

Ejemplo 4.5 ((Leibniz). Expresar $y=\operatorname{sen} \theta$ como una serie infinita). Solución. Sea un cuarto de circunferencia unitaria, $P=(x, y)$ un punto sobre ella y $\theta$ el ángulo formado por BOP; consideremos el triángulo rectángulo infinitesimal con catetos $d x, d y$ e hipotenusa $d \theta$, que es semejante con el triángulo PDO.

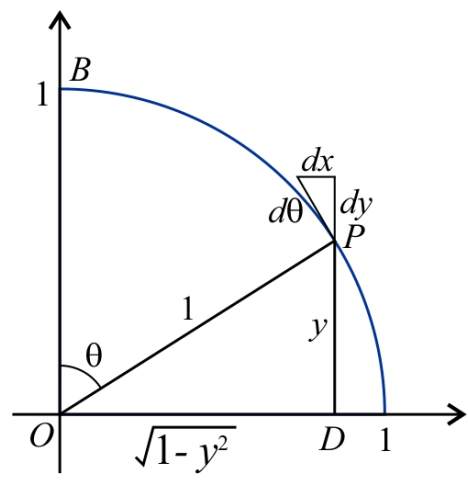

Figura 4.4: Desarrollo de $\operatorname{sen}(\theta)$ en una serie infinita

Luego se tiene,

$$
\frac{d x}{d y}=\frac{y}{\sqrt{1-y^{2}}} \cdot \therefore \frac{d x^{2}}{d y^{2}}=\frac{y^{2}}{1-y^{2}} .
$$

Por Pitágoras $d x^{2}+d y^{2}=d \theta^{2}$, pero $d x^{2}=\frac{y^{2} d y^{2}}{1-y^{2}}$, entonces

$\frac{y^{2} d y^{2}}{1-y^{2}}+d y^{2}=d \theta^{2}$, de donde $\frac{1}{1-y^{2}} d y^{2}=d \theta^{2}$, ó $d y^{2}+y^{2} d \theta^{2}=d \theta^{2}$, (*) la que es una ecuación diferencial. $y=\operatorname{sen} \theta$ es la solución. En efecto, $d y^{2}=\cos ^{2} \theta d \theta^{2}$, luego $\cos ^{2} \theta d \theta^{2}+\operatorname{sen}^{2} \theta d \theta^{2}=d \theta^{2}$.

¿Cómo resolvió Leibniz la ecuación diferencial (*)? El considera a d $\theta$ como una constante, luego considera al operador diferencial d y sus reglas de operar; aplica d a ambos miembros de (*) y se obtiene $d\left(d y^{2}+\right.$ $\left.y^{2} d \theta^{2}\right)=0$, de donde $2 d y d d y+2 y d y d \theta^{2}=0$, ó $d y d^{2} y+y d y d \theta^{2}=0$ ó $d^{2} y+y d \theta^{2}=0$ ó $\frac{d^{2} y}{d \theta^{2}}=-y$, una ecuación que tiene por solución a $y=\operatorname{sen} \theta$. Leibniz supone que podemos representar al seno como una serie de potencias con coeficientes indeterminados en la forma $y=\operatorname{sen} \theta=a_{1} \theta+a_{3} \theta^{3}+a_{5} \theta^{5}+$ $a_{7} \theta^{7}+a_{9} \theta^{9}+\ldots$, donde $a_{o}=0$ pues sen $0=0$ y solo se tienen potencias impares pues sen $\theta$ es impar.

Derivando con respecto a $\theta$ tenemos $\frac{d y}{d \theta}=a_{1}+3 a_{3} \theta^{2}+5 a_{5} \theta^{4}+7 a_{7} \theta^{6}+\ldots y \frac{d^{2} y}{d \theta^{2}}=2 \cdot 3 a_{3} \theta+4 \cdot 5 a_{5} \theta^{3}+$ $6 \cdot 7 a_{7} \theta^{5}+\ldots$.

Reemplazando estas expresiones en $\frac{d^{2} y}{d \theta^{2}}=-y$ se obtiene:

$2 \cdot 3 a_{3} \theta+4 \cdot 5 a_{5} \theta^{3}+6 \cdot 7 a_{7} \theta^{5}+\ldots=-a_{1} \theta-a_{3} \theta^{3}-a_{5} \theta^{5}-\ldots$

Igualando los respectivos coeficientes, se tiene: $2 \cdot 3 a_{3}=-a_{1}, 4 \cdot 5 a_{5}=-a_{3}, 6 \cdot 7 a_{7}=-a_{5}, \ldots$

Tomando $a_{1}=1$ se obtiene:

$a_{3}=-\frac{1}{2 \cdot 3}=-\frac{1}{3 !} ; a_{5}=-\frac{1}{4 \cdot 5} a_{3}=-\frac{1}{4 \cdot 5}\left(-\frac{1}{3 !}\right)=\frac{1}{5 !} ; a_{7}=\frac{1}{6 \cdot 7}\left(-\frac{1}{5 !}\right)=-\frac{1}{7 !}, \ldots$ 
Por lo tanto, $y=\operatorname{sen} \theta=\theta-\frac{1}{3 !} \theta^{3}+\frac{1}{5 !} \theta^{5}-\frac{1}{7 !} \theta^{7}+\ldots$.

Nota. Esta representación de $y=\operatorname{sen} \theta$ fue también obtenida por Newton.

ORCID and License

Alejandro Ortiz Fernández https: / / orcid.org/0000-0002-9380-4301

This work is licensed under the Creative Commons - Attribution 4.0 International (CC BY 4.0)

\section{Referencias}

[1] Bourbaki N. Elementos de Historia de las Matemáticas. Alianza Editorial. Hermann. París. 1969.

[2] Dunham W. Journey Through Genius. John Willey.N.Y. 1990.

[3] Gonzales P. Arquímedes y los Orígenes del Cálculo Integral. Nivola. 29. España. 2008.

[4] Gonzales P. Orígenes y Evolución Histórica del Cálculo Infinitesimal [Internet] disponible en http://www.ugr.es/dpto_am/OLD/docencia/Apuntes/Origenes_del_Calculo.pdf.

[5] Grattan-Guinness I. From the Calculus to Set Theory, 1630-1910. New York: Princeton University Press. 1980.

[6] Heath TL. The Works of Archimedes. Cambridge: Cambridge University Press. 1897.

[7] Ortiz A. La Matemática a Través de Clásicas Áreas. Lima: Vol 2. Public Digital. PUCP; 2016.

[8] Rey Pastor J., Babini J. Historia de la Matemática. Buenos Aires: Espasa-Calpe; 1951. 